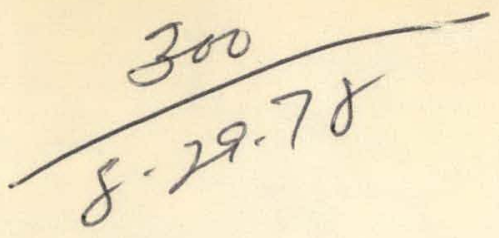

Assistant Secretary for Energy Technology

Division of Solar Energy

Under Contract No. EG-77-C-01-4101

\title{
Biomass Fuel Systems \\ Directory of Sources and Potential Users
}




\section{DISCLAIMER}

This report was prepared as an account of work sponsored by an agency of the United States Government. Neither the United States Government nor any agency Thereof, nor any of their employees, makes any warranty, express or implied, or assumes any legal liability or responsibility for the accuracy, completeness, or usefulness of any information, apparatus, product, or process disclosed, or represents that its use would not infringe privately owned rights. Reference herein to any specific commercial product, process, or service by trade name, trademark, manufacturer, or otherwise does not necessarily constitute or imply its endorsement, recommendation, or favoring by the United States Government or any agency thereof. The views and opinions of authors expressed herein do not necessarily state or reflect those of the United States Government or any agency thereof. 


\section{DISCLAIMER}

Portions of this document may be illegible in electronic image products. Images are produced from the best available original document. 
Available from:

National Technical Information Service (NTIS)

U.S. Department of Commerce

5285 Port Royal Road

Springfield, Virginia 22161

Price: Printed copy: $\$ 6.50$

Microfiche: $\$ 3.00$ 
HCP/T4101-04

UC-61
Prepared for U.S. Department of Energy

Assistant Secretary for Energy Technology

Division of Solar Energy

Fuels from Biomass Systems Branch

Washington, D.C. 20545

\section{Biomass Fuel Systems}

Directory of Sources and Potential Users

Prepared by

J.F. Henry

D.J.Salo

M.S. Schauffler

B.T. Smith

Metrek Division

of MITRE Corporation

McLean, Virginia 22101

Under Contract No. EG-77-C-01-4101 


\section{NOTICE}

This report was prepared as an account of work sponsored by the United States Government. Neither the United States nor the United States Department of Energy, nor any of their employees, makes any warranty, express or implied, or assumes any legal liability or

responsibility for the accuracy, completeness, or usefulness of any information, apparatus, product, or process disclosed, or represents that its use would not infringe privately owned rights. Reference herein to any specific commercial product, process, or service by trade name, mark, manufacturer, or otherwise, does not necessarily constitute or imply its endorsement, recommendation, or favoring by the United States Government or any agency thereof. The views and opinions of authors expressed herein do not necessarily state or

reflect those of the United States Government or any agency thereof. 


\section{ABSTRACT}

Sources and potential users of technical information on biomass fuel systems have been identifled. Organizations and individual contacts are listed in various production and conversion categories. 


\section{THIS PAGE \\ WAS INTENTIONALLY \\ LEFT BLANK}


TABLE OF CONTENTS

$\underline{\text { Page }}$

INTRODUCTION

ORGANIZATION OF THE INFORMATION BASE

I. SOURCES OF INFORMATION 11

A. GENERAL INFORMATION 12

B. SYSTEMS STUDIES 13

$\begin{array}{ll}\text { C. BIOMASS PRODUCTION } & 16\end{array}$

1. Residues . 16

a. Forest Residues 16

b. Agricultural-Residues 19

2. Energy Farms 21

a. Silvicultural Energy Farms 21

Biomass Harvesting Equipment $\quad 27$

b. Agricultura1 Energy Farms 28

c. Fresh Water Energy Farms 29

d. Marine Energy Farms ' 30

D. CONVERSION TECHNOLOGIES 35

1. Densification 33

2. Thermochemical Processes 35

a. Direct Combustion 35

Architecture and Engineering Firms. 39

b. Gasification/Pyrolysis 40

c. Liquefaction/Pyrolysis 43

3. Bioconversion Processes 44

a. Fermentation 44

h. Anaernhic Digestion 47 
TABLE OF CONTENTS (Concluded)

II. RECIPIENT'S OF INFORMATION

A. FEDERAL AGENCIES

B. STATE AND REgIONAL AGENCIES 55

C. TRADE ASSOCIATIONS 65

D. UTILITIES $\quad 70$

E. INDUSTRY $\quad 71$

1. Forest Products 71

2. Petrochemicals 74

3. Auto/Petroleum 75

4. Wood, Boller Firms 76

5. Other Industries 82

F. AGRICULTURE $\quad 83$

1. Producers/Processors 83

2. Machinery/Manufacturers : 85

G. ALTERNATIVE ENERGY INFORMATION ORGANIZATIONS 86

H. INTERNATIONAL ORGANIZATIONS 88

INDEX $\quad 89$ 


\section{J.NTRODUCTION}

Biomass is receiving considerable attention as an important fuel and chemical feedstock. As a result, the Department of Energy has received numerous requests for technical information on biomass fue1 systems.

It would be impossible to obtain detailed information about all aspects of "blomass fuels" from a single source because so many production, conversion and utflization options exist. The best information can be obtained from those organizations which are actively involved in the development, design, manufacture and use of specific biomass fuel systems. Many of these sources of information are identified here. Representative users of technical information are also listed.

Sources of technical information on biomass fuel systems are identified in Section I. These sources are grouped under general headings such as biomass production and conversion technologies These general categories are futher divided, whenever possible, to Identify specific biomass feedstocks and conversion systems.

A partial 1ist of potential users of technical information is presented in Section II. This 1ist includes organizations, such as forest product companies, which are obviously interested in the development of biomass fuel systems. It also includes groups which 
are showing a new awareness of the potential uses of biomass energy. The National Realtors Association and the National Recreation and Park Association are representative of this group. In many cases, sources of technical information are also users of that information. Placement of an organization into one category or subcategory rather than another was of ten based on an arbitrary. division. An alphabetical index is provided to help locate specific references.

It is necessary to emphasize that "fuels from biomass" is a rapldiy changing field. New approaches and technological advances can be expected to change biomass R\&D and commercialization programs. It will be necessary to frequently revise this document if it is to reflect the current "state-of-the-art." 
ORGANIZATION OF THE INFORMATION BASE

The directory is divided into two general categories. (See Table I, following page.) Category I contains the names of persons and organizations which are informed on what is happening in a particular area of bio-energy development. Category II is a sampling of companies, government agencies and other organizations which may be interested in receiving current data on bio-energy research and development.

The line between a source of information and a potential user is a fine one. In many cases an organization could appropriately be listed in both categories. The names which appear in the second category represent for the most part some of the industries and organizations which have shown specific interest in biomass in recent years.

The general organization of the information base in Category I can be summarized as follows:

A. General Information - This category includes those organizations capable of giving an overview of biomass as a fuel or energy resource, and of orienting a potential user towards more specific information relating to his area of interest.

B. Systems Studies - This category includes those organizations which have conducted systems engineering studies, resource recovery analyses and conceptual research relating to the use of biomass as fuel. Where posstble the major area of involvement is indicated. 
TABLE I

SOURCES AND RECIPIENTS OF TECHNICAL INFORMATION ON BIOMASS FUEL SYSTEMS

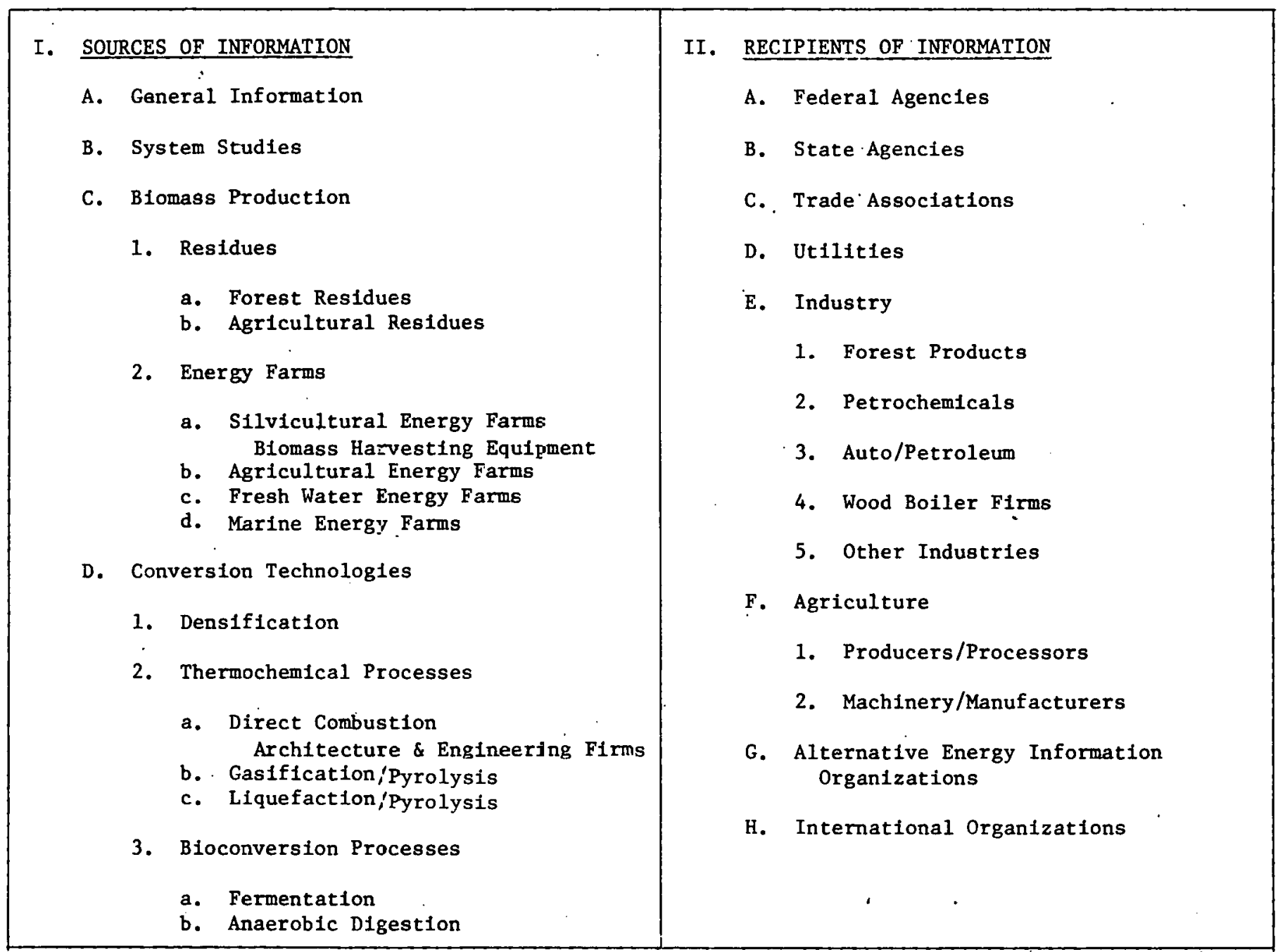


C. Biomass Production - This category includes organizations which are involved in research and development or in commercialization relating to production of biomass. Subcategorles within the production area are as follows:

1. Residues

a. Forest Residues - These include biomass materlal associated with the forest industry operations, logging and mill residues. Surplus forest growth is also considered a residue. This source is presently available and equipment exists to recover it. Improved collection machinery is presently being developed. Within certain climatic limitations this blomass resource is available on a year-round basis.

b. Agricultural Residues - This resource includes crops and feed-lot manures. The avallability of crop biomass generally follows a seasonal pattern, but manures are avaflable on a year-round basis. Collection systems for agricultural residues are well-developed but improved methods, including on-site densification, are being researched.

2. Energy Farms

a. Silvicultural Energy Farms - Tree crops will be intensively managed on a short rotation schedule. The technology of tree farming will be based on agricultural practices such as irrigation and fertilization. Continuing research includes identifying and developing high-yield species. This resource is available on a rotating year-round basis.

Companies involved in the manufacture and/or development of specialized tree harvesting equipment are listed in this section under "Biomass Harvesting Equipment".

b. Agricultural Energy Farms - This resource involves the production of agricultrual crops such as wheat, corn, sugarcane, sorghum, etc., and other herbaceous plants such as grasses for their fuel rather than food value. Conventional production and 
harvesting technology is well established. In most areas this resource will be available on a seasonal basis.

c. Fresh Water Energy Farms - Fresh water plants such as various algae, duckweed and water hyacinth can be grown for their fuel value. Research and development has begun on improving production and harvesting systems.

d. Marine Energy Farms - Intensive research is underway to develop certain marine plants, particulariy giant California kelp, as a food/fuel/feedstock resource. This source would be available on a year-round basis.

D. Conversion Technologiés

1. Densification

Densification or pelletization is a method for converting biomass into a homogeneous, easily handled and stored organic fuel. Densified biomass can be used in direct combustion systems or as a feedstock for thermochemical conversion. The technology is presently available and can be used on a wide variety of biomass materials.

2. Thermochemical Processes

a. Direct Combustion - Direct Combustion of biomass to produce steam/heat or electricity is a well established technology. An increasing number of users are relying on direct combustion to supply a part or all of their energy needs. A separate heading, "Architecture and Engineering Firms" is included at the end of this section. These firms are specifically knowledgable in wood-fired combustion systems.

- b. Gasification/Pyrolysis - Numerous gasification systems using biomass materials are in the process of development. Some experimental and commercial gasification units are already in production.

c. Liguefaction/Pyrolysis - This technology is still in the experimental stage. Most of the sources listed are, therefore, research and development programs. Where possible the source of biomass feedstock is identified. 


\section{Bioconversion Processes}

a. Fermentation - Source data in this section includes research into the hydrolysis of cellulosic material and the fermentation of sugars to alcohol. Fermentation is a well-established technology, but research is being directed to the improved conversion of cellulosic materials to alcohols.

b. Anaerobic Digestion - Anaerobic digestion is the result of bacterial conversion of biomass to methane in the absence of oxygen. The technology involved is currently available for use with certain biomass feedstocks such as manures and certain cellulosic residues. Improved techniques are being developed in current research programs.

Category II is defined as Recipients of Information and poten-

tial users of biomass. Many names listed in this category are also

excellent sources of information in specific biomass areas.

A. Federal Agencies - Many federal agencies and institutions should receive up-to-date information on biomass systems. Some of the agencies which have expressed interest in bio-energy are listed here.

B. State Agencies - State agencies, commissions or offices should be recipients of biomass data. Often they are good sources of local biomass developments.

r. Trade Assoniations - Natinnal trade and professinnal associations are a good way to disseminate technical information to a large audience. Included in this section is a partial listing of associations considered to be potentfally interested recipients of technical information on biomass development. A complete list of trade associations can be found in the directory: National Trade and Professional Associations of the United States and Canada and Labor Unions, 13th annual edition, 1978, Craig Colgate, Jr., editor. Columbia Books, Inc., Washington, DC 20005.

D. Utilities - Some of the utilities which have expressed an interest in bio-energy development and application are included in this section. Additional utility companies can be identified in the trade association list. 
E. Industry - Because of the nature of their operations some industries may be more interested in bioenergy development for fuel than others. This section has therefore been divided into subcategories which identify some companies presently using biomass systems or which have expressed interest in such systems. The list is by no means complete, and can be expanded by referring to the section on trade associations.

\section{Forest Products}

Companies interested in the use of biomass for process steam/heat generation. Many of the firms listed are also potential sources of data on biomass production, recovery and handling.

2. Petrochemicals

Companies interested in biomass as a feedstock to produce chemicals and substitute fuels.

3. Auto/Petroleum

Companies interested in biomass-derived transportation fuels.

4. Wood Boiler Firms

Companies interested in the market resulting from an increased use of biomass as fuel. Some of the companies listed here should also be considered as sources of information on direct combustion.

5. Other industries

Miscellaneous companies which have expressed some interest in biomass as a fuel or feedstock.

\section{F. Agriculture}

1. Producers/Processors

Companies which have expressed interest in the use of agricultural surplus production and residues from both production and processing as a source of energy or feedstock are included 


\section{Machinery Manufacturers}

Companies which are involved in the harvesting or densification of agricultural materials and have shown interest in bio-energy are included.

G.' Alternative Energy Information Organizations - The organizations listed here represent a limited sampling of citizen and consumer groups as well as some public-interest research groups which are involved in alternative energy dissemination.

H. International Organizations - Many of the organizations ilsted are experts on bio-energy programs being developed in other countries and are prime candidates for information exchange. 


\section{THIS PAGE}

\section{WAS INTENTIONALLY LEFT BLANK}


I. SOURCES OF INFORMATION 


\section{SOURCES OF INFORMATION}

\section{A. General Information}

The Bio-Energy Council

1337 Connecticut Avenue, NW

Washington, DC 20036

Pau1 Bente

(202) 833-5656

The Biomass Energy Institute 304-870 Cambridge Street Winnipeg, Manitoba R3M 3H5, Canada

Ernest E. Robertson

(204) 284-0472

Energy, Department of

Fuels From Biomass Branch

600 E street

Washington, DC 20545

Nello delGobbo

Acting Chief

(202) $376-1610$

Oak Ridge National Laboratory

P.O. Box X, Building 2024

Oak Ridge, TN 37380

Robert VanHook

(615) 483-8611

Solar Energy Research Institute

Research Division, Biological and Chemical Conversion Branch 1536 Cole Boulevard

Golden, CO 80401

T. A. Milne, Acting Chief (303) $234-7171$

Tennessee Valley Authority

Commerce Union Bank Building

Chattanooga, TN 37400

Harold Falkenberry

(615) $755-3011$ 


\section{B. Systems Studies}

Joseph E. Atchison, Inc.

30 East 42 nd $S t$.

New York, NY 10017

Joseph E. Atchison

(2.12) 986-0016

Subject: Preliminary Evaluation of New Process for Separation of Components of Sugar Cane

Battelle Memorial Laboratories

505 King Avenue

Columbus, OH. 43201

Edward S. Lipinsky

(614) 424-6424

Subject: System Studies of Fuels from Sugar and Corn

California Institute of Technology

Jet Propulsion Laboratory

4800 Oak Grove Drive

Pasadena, CA 91103

J. J. Kalvinskas

(213) 354-2349

Subject: Technical and Economic Evaluation of Bioconversion Processes as Alternative Source of Energy

Dartmouth College

Power Task Force

Box 8000

Hanover, NH 03755

Dennis Meadows

(603) 646-3551

Subject: Identify Social, Economic and Environmental Impacts of Wood Burning in the Northeast

Development Planning \& Research Assoclaces, Inc. 200 Research Drive, P.0. Box 727

Manhaltan, KS 66502

(913) 539-3565

Subject: Gasohol Economic Feasibility Study 
B. Systems Studies (Continued)

Dynatech R\&D Company

99 Erie Street

Cambridge, MA 02139

Donald L. Wise

(617) 868-8050

Subject: Fuel Gas Production from Animal

Residue; Aquatic Biomass Farms

Institute of Gas Technology

3424 South State Street

Chicago, IL 60610

Donald Klass

(312) $567-3650$

Subject: Gasification and Fermentation Studies Using Various Biomass Sources

InterTechnology/Solar Corporation

Box 340

Warrenton, VA 22186

G. C. Szego

(703) 347-7900

Subject: Integrated Energy Farms

Midwest Research Institute

425 Volker Boulevard

Kansas C1cy, MÜ 64110

A. D. McE1roy

(816) 753-7600

Subject: Systems Study of Fuels from Grains

The MITRE Corporat1on/Metrek Division

1820 Dolley Madison Boulevard

McLean, VA 22102

David J. Salo

(703) 827-6000

Subject: Silvicultural Biomass Study

North Carolina State University

School of Forest Resources

Raleigh, NC 27650

M. P. Levi

(919) 737-3386

Subject: Economics and Characteristics of Wood Handling Systems in Small to Medium

Sized Boilers 
B. Systems Studies (Concluded)

TEKNEKRON

2118 Milvia Street

Berkeley, CA 94704

Gladys Sessler

(415) 848-1464

Subject: Agricultural Crops for Fuel

United Technologies Research Center

Silver Lane

East Hartford, CT 06108

Robert Lessard

(203) 565-4321

Suhjert: Assessment nf Rinmass Potential for

Electric Power Generation in the

Northeast 
C. Biomass Production

1. Residues .

a. Forest Residues

Complete Tree Institute

University of Maine

Orono, ME 04473

Harold E. Young

(207) $581-1110$

Subject: Wood as Fue1

WESTVACO Research Center

P.0. Box 5207

N. Charleston, SC 29406

S. Ingemar Falkehag

(803) 744-8231

Subject: Pulping and Lignocellulose Ut1lization

Weyerhaueser Company

Tacoma, WA 98401

Robert L. Jamison

(206) 924-2697

Subject: Develop Maximum Use of

Forest Residues as Energy

Source

Forest Service Field Offices:

Forest Service, USDA

Northern Region ( $R-1)$

Federal Bullding

Missoula, MO 58907

Forest Service, USDA

Rocky Mountain Region (R-2)

11177 West 8 th Avenue

P.O. Box 25127

Lakewood, CO 80225

Forest Service, USDA

Southwestern Region ( $R-3)$

Federal Building

517 Gold Avenue, SW

Albuquerque, NM 87102 


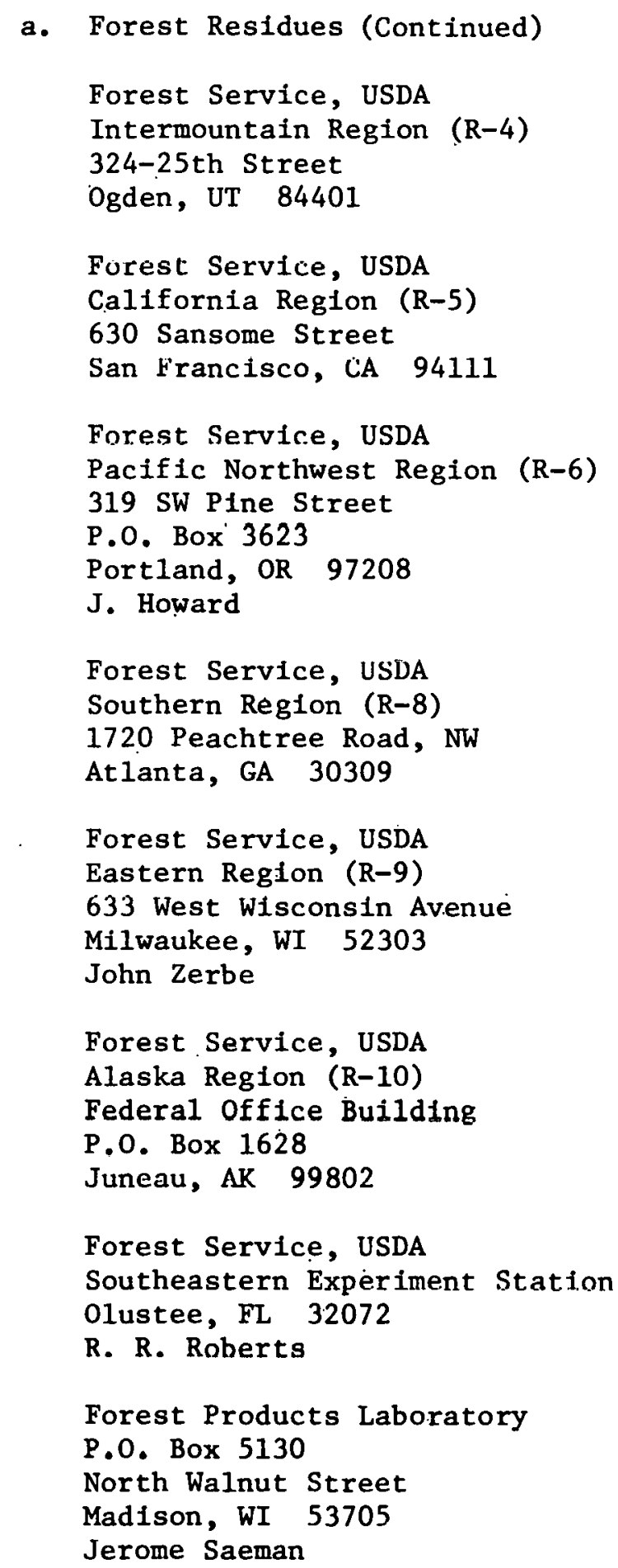


a. Forest Residues (Concluded)

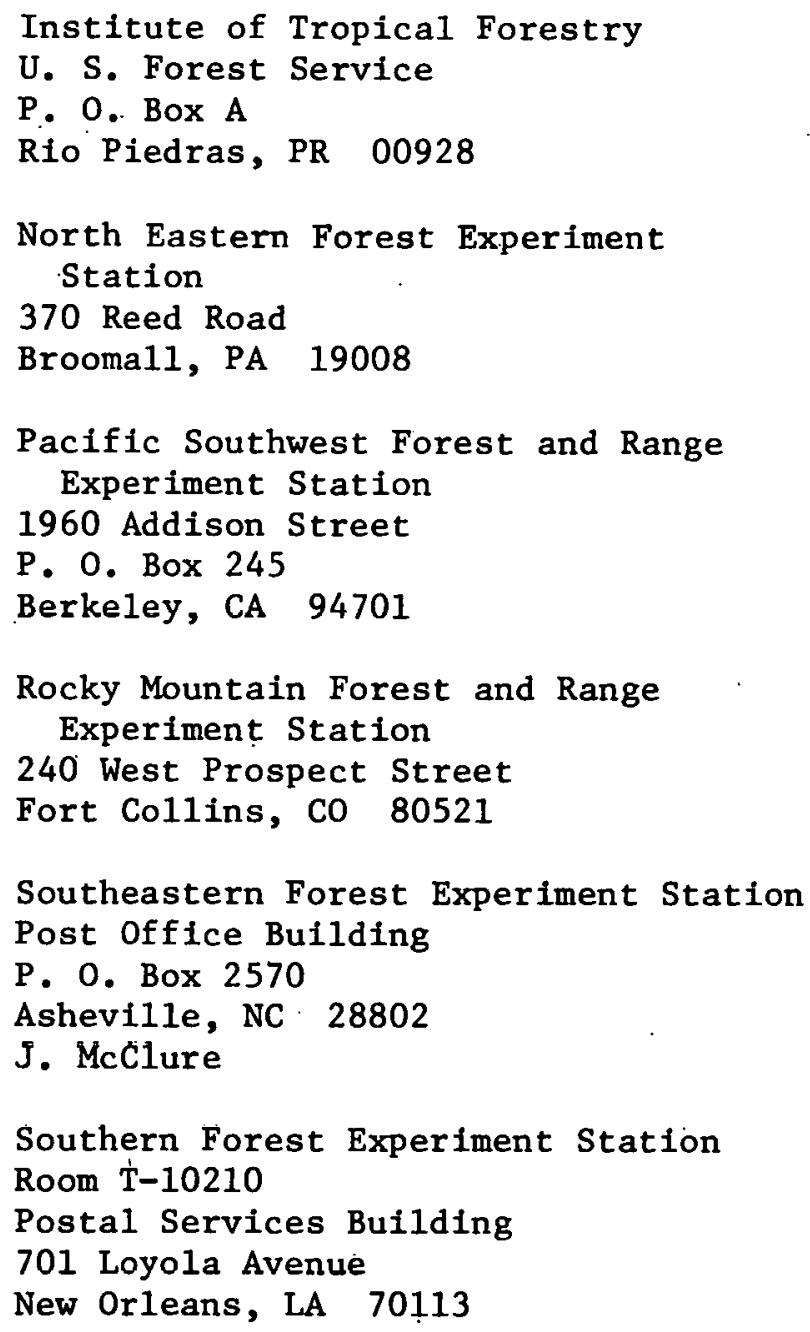


b. Agricultural Residues

Hawaiian Sugar Planters Association

P.0. Box 1057

Aiea, Oahu, HI 96701

Edward J. Lui

(808) 487-5561

Subject: Research on Sugar Crops as

Energy Biomass

Oahu Sugar Company, Ltd.

Waipahu, Oahu, HI 96797

R. Proffitt

(808) 677-3357

Subject: Sugarcane Residues for Cogeneration in Mill Operations

Stanford Research Institute

333 Ravenswood Avenue

Menlo Park, CA 94025

(415) 326-6200

Subject: Evaluation of Agricultural

Residues of Energy Feedstock

University of California

Lawrence Berkeley Laboratory

Laboratory of Chemical Biodynamics

Berkeley, CA 94700

Melvin Calvin

(514) 843-2740

Subject: Examination of Photosynthesis as a Possible Renewable Energy Source

University of Florida

Agricultural Research Center

Belle Glade, FL 33430

Gary L. Gascho

(305) 996-3062

Subject: Sugar Cane Biomass Production

USDA/AES

University of Missouri

Columbia, MO 65201

Elmer R. Kieh1

(314) 882-3846

Subject: Food, Fiber and Energy 
b. Agricultural Resldues (Concluded)

USDA/ARS

U.S. Sugarcane Field Laboratory

P. O. Box 470

Houma, LA 70361

James E. Irvine

(504) 872-6316

Subject: Close Row Spacing for Blomass

Production 


\section{Energy Farms}

a. Silvicultural Energy Farms

Agricultural Experiment Station

University of Kentucky

Lexington, KY 40506

S. B. Carpenter

(606) 258-4609

Department of Forestry

Illinois Agricultural Exp. Sta.

University of Illinois

Urbana, IL 61801

F. I. RnIfe

(217) 333-1000

Subject: Biomass Production for Energy Conviersion

Department of Forestry

Iowa State University

Ames, IA 50010

J. Gordon

(515) 294-1166

Subject: Yield Prediction of Hardwoods

Department of Forestry

Kansas State University

Manhattan, KS 66506

W. A. Geyer

(913) 532-6923

Subject: Sycamore, Maple, Cottonwood

Department of Forestry

Michigan Technological University

Houghton, MI 49931

R. L. Sajdak

(906) 487-1885

Subject: Sugar Maple and Black Locust to Energy Farm Concept

Georgfa Institute of Technology

Atlanta, GA $30332^{\circ}$

Daniel O'Neil

(404) 894-2000

Subject: Production of Trees for Fuels 


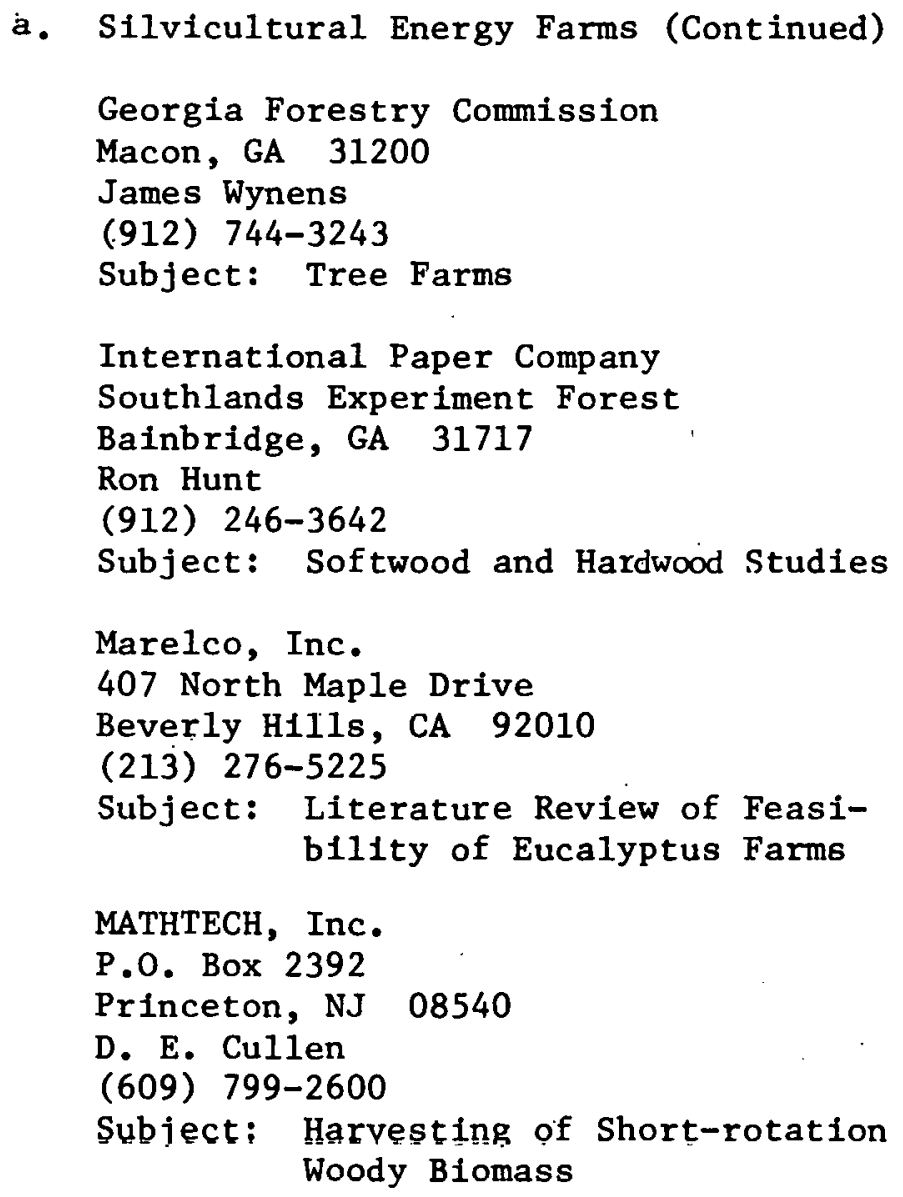




\author{
a. Silvicultural Energy Farms (Continued) \\ North Carolina State Unfversity \\ Department of Forestry \\ Raleigh, NC 27607 \\ Robert Kellison \\ (919) 953-3717 \\ Subject: Tree Farm Research \\ Pennsylvania State University \\ University Park, PA 16802 \\ T. W. Bowersox \\ (814) 865-4841 \\ Subject: Hybrid Poplars \\ Pennsylvania State University \\ University Park, PA 16802 \\ W. E. Sopper \\ (814) 865-2541 \\ Subject: Biomass Production Using \\ Waste Heat \\ School of Forest Resources \\ North Carolina State University \\ Raleigh, NC 27650 \\ M. P. Levi \\ (919) 737-3386 \\ Subject: Wood Fuel Handling in Small/ \\ Medium Boilers \\ School of Forest Resources and Conser- \\ vation \\ University of Florida \\ Galnesville, FL 32611 \\ George Meskimen \\ (904) 392-1971 \\ Subject: Eucalyptus Energy Farms \\ South Carolina Commission of Forestry \\ P.U. Box $2170 \%$ \\ Columbia, SC 29200 \\ Marvin Gaffney \\ (803) 758-2261 \\ Subject: Tree Energy Farms
}




\section{a. Silvicultural Energy Farms (Continued) \\ Seattle City Light Company \\ Office of Environmental Affairs \\ 1015 Third Avenue \\ Seattle, WA 98104 \\ C. S. Harlow. \\ (206) 625-3590 \\ Subject: Forest Production for Fuel}

Solar Energy Research Institute

1536 Cole Boulevard

Golden, Co 80401

Robert Inman

(303) 234-7171

University of California-Riverside

Dept. of Soil \& Environmental Sciences

Riverside, CA 92521

P. Felker

(714) 787-3706

Subject: Species Growth Rate Under Controlled Conditions

University of Georgia

School of Forest Resources

Athens, GA 30602.

Klaus Steinbeck

(404) 542-1376

Subject: Hardwond Production

University of Houston

3800 Cullen Boulevard

Houston, TX 77004

J. R. Cowles

(713) 749-3135

Subject: Chinese Tallow Tree as Biomass

Fue1 Source 
a. Silvicultural Energy Farms (Continued)

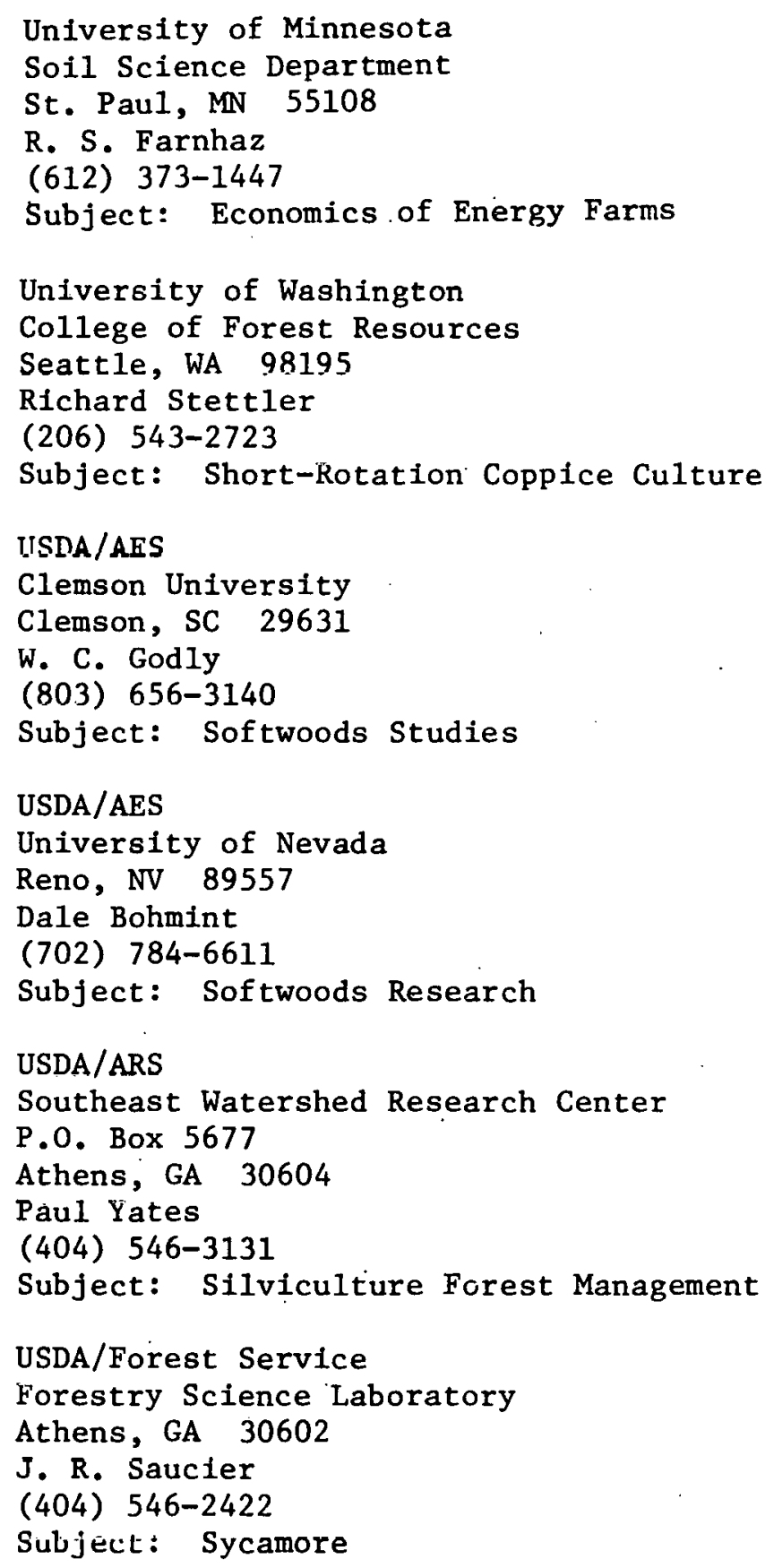


a. Silviculturai Energy Farms (Continued)

USDA/Forest Service

North Central Experiment Station

Box 898

Rhinelander, WI 54501

David L. Dawson

(715) 362-7474

Subject: Intensive Biomass-Yield Tree Farms

USDA/Forest Service

Southeastern Forest Experiment Station P.0. Box 2570

Asheville, NC 28802

Jack Stubbs

(803) 824-6331

Subject: Research on Paraquat-Treated

Pine Trees

Utah State University

Institute for Land Rehabilitation

Logan, UT 84321

C. M. McKel1

(801) 752-4100

Subject: High Producing Shrubs for Blomass

Virginia Polytechnic Institute

Blacksburg, VA 24061

T. A. Walbridge

(703) 951-6391

Subject: Investigation of Woody Biomass Systems

Virginia State Forestry Commission

Charlottesville, VA 22901

Wallace F. Custard

(804) 977-6555

Subject: Research on Energy Tree Farms

Washington State University

Pullman, WA 99164

R. C. Chapman

(509) 335-5296

Subject: Evaluation of Tree Blomass Yields 
a. Silvicultural Energy Farms (Concluded)

Biomass Harvesting Equipment

Morbark Industries

Winn, MI 48896

Pete Ratcliffe

(517) 866-2381

Subject: Harvesting Equipment

N. F. I., Inc:

6407 Masonic Drive

Alexandria, LA 91301

James O'Dair

(318) 487-8371

Nicholson Manufacturing Company

$3670 \mathrm{E}$. Marginal Way, S.

Seattle, WA 98134

W. T. Nicholson

(206) 682-2752

North Carolina Forest Extension

Service

James Mattson

Houghton, MI 49931

Subject: Biomass Harvesters

Rome Industries

Cedartown, GA 30125

Sam Coughran

Subject: Tree Puller that Harvests

Central Roul Mass

USDA/FS

Southern Forest Experiment Station

2500 Shreveport Highway

Pineville, LA 71360

Peter Koch

(318) $445-6511$

Subject: Reference Information and

Biomase Harvecter 
b. Agricultural Energy Farms

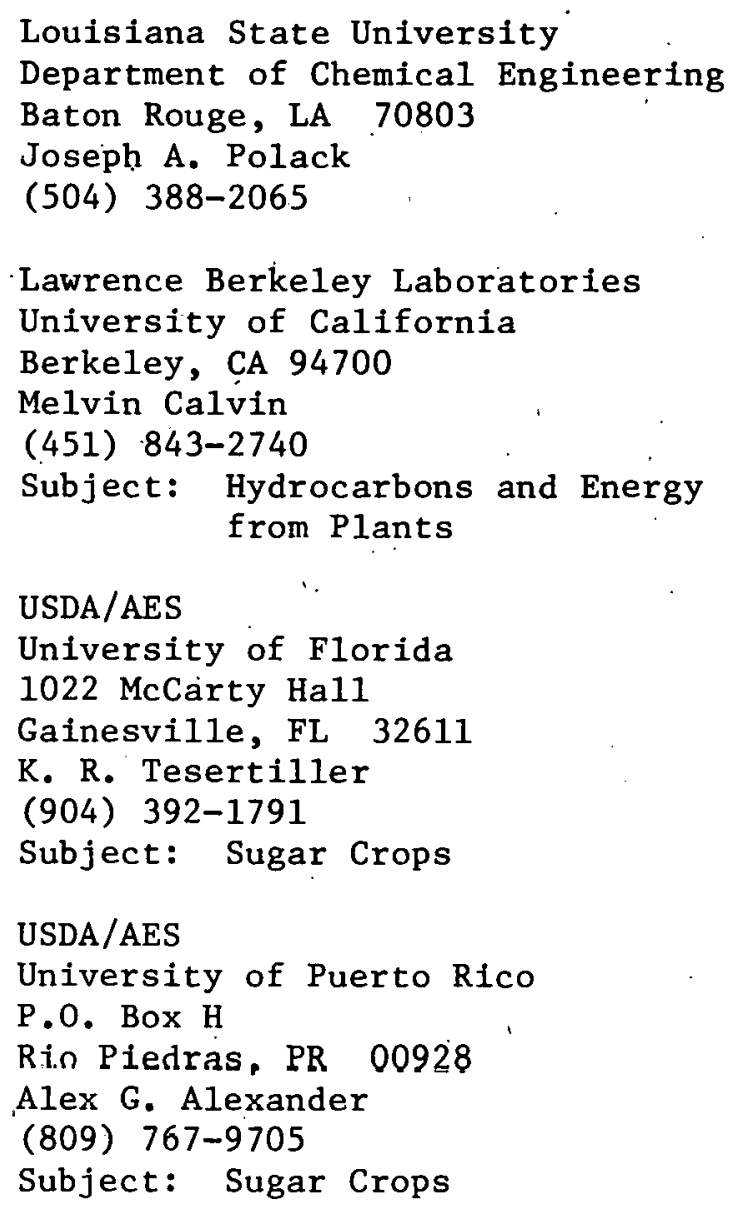




\section{c. Fresh Water Energy Farms \\ Battelle Columbus Laboratories \\ 505 King Avenue}

Columbus, $\mathrm{OH} \quad 43201$

A. C. Robinson

(614) 424-6424

Subject: Hyacinth Research

University of California

College of Engineering

301 S 46th Street

Richmond, CA 94804

W. J. Oswald

(415) 642-0835

Subject: Algae Research

University of California

Lawrence Livermore Laboratory

Livermore, CA 94550

Raymond L. Ward

(415) 457-4976

Subject: Algae Studies

University of Manitoba

Department of Botany

Winnipeg, Manttoba

Canada R3T 2N2

E. Roy Waygood

(204) 474-8880

Subject: Algae Production on Waste Water

National Aeronautics and Space Administration

NSTL Station

Bay St. L ouis, MS 39529

B. C. Wolverton

(601) 688-3155

Subject: Water Hyacinth Plant Program 


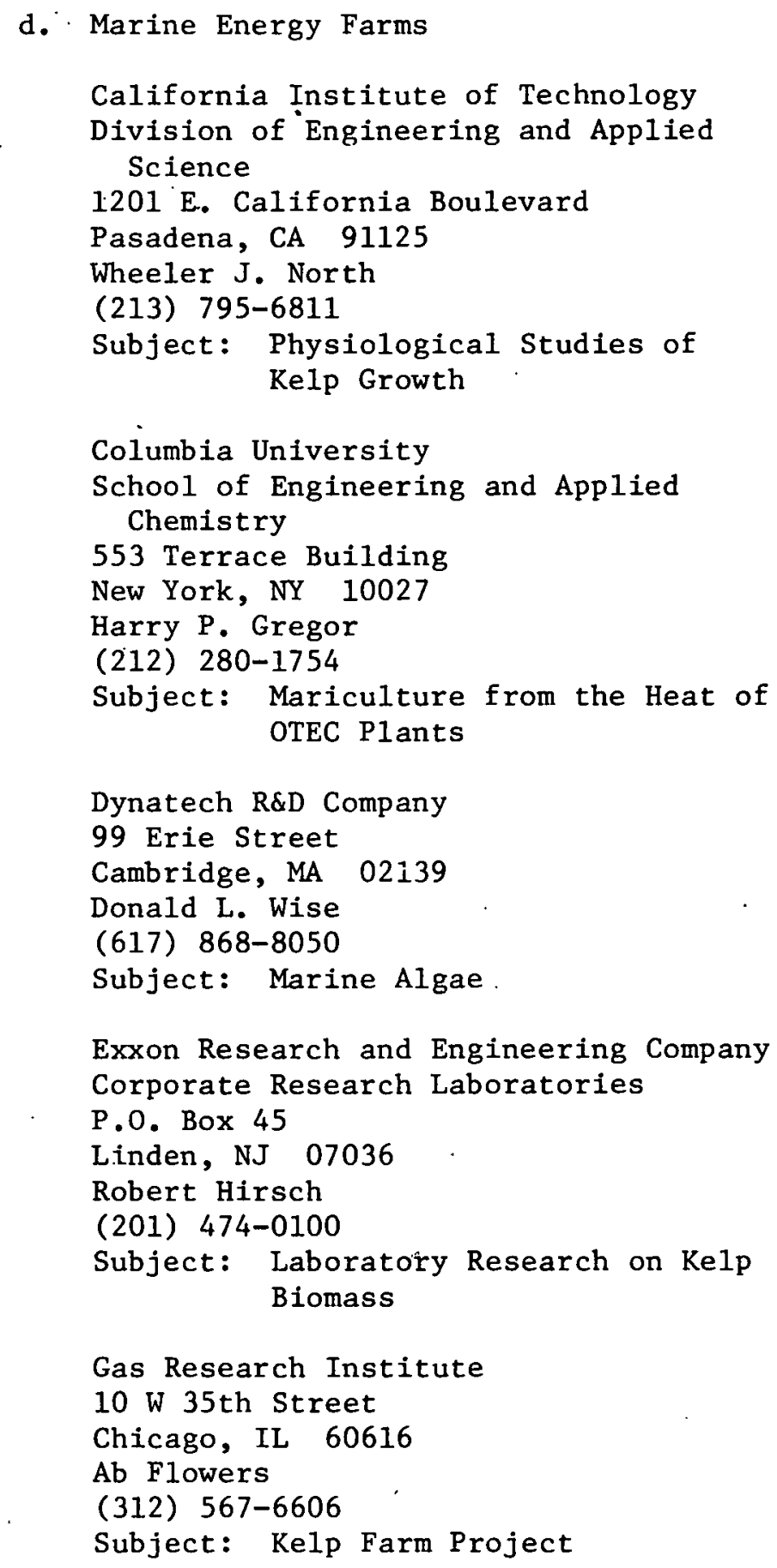


d. Marine Energy Farms (Continued)

General Electric Company

King of Prussia, PA 19406

Alan. Tompkins

(215) 962-6000

Subject: Kelp Farm Project

General Electric Company

777 14th Street NW

Washington, DC

Rudolf Trefney

(202) 637-4000

Subject: Marine Bioconversion

Froducelon

Global Marine Development, Inc, Newport Beach, CA 92600

Manfred Kruteen

(714) 752-5050

Subject: Kelp Farm Project

Groton BioIndustries Development

Company

Division of Groton Associates, Inc.

P. 0. Box 517

Groton, MA 01450

Harold H. Webber

(617) 448-5916

Subject: Design \& Implementation of Aquaculture Systems

Nava1 Ocean Systems Center

San Diego, CA 92132

Howard Wilcox

(714) 225-2354

Subject: Production and Conversion of Ocean Energy Farm Systems

Science Applications, Inc.

1200 Prospect Street

LaJolla, CA 92038

Gilbert R. Stegen

(714) 459-0211

Subject: Assessment of Marine Biomass

Materials 


\section{d. Marine Energy Farms (Concluded)}

Woods Hole Oceanographic Institute Woods Hole, MA 02543

John H. Ryther

(617) 548-1400

Subject: Potential of Aquatic Plants

as' Biomass Source 
D. Conversion Technologies

1. Densification

Agnew. Environmental Products

211 SE 10th Street

P.0. Box 1168

Grants Pass, OR 97526

L. L. Thompson

(503) 479-3396

Bio-Solar Research \& Development Corporation

P.O. Box 762

Eugente, OR 97401

Rudolf W. Gunnerman

(503) 686-0765

Bonnot Company

805 Lake Street

Kent, OH 44240

(216) 673-5829

California Pellet Mill Company 1800 Folsom Street

San Francisco, CA 94013

Robert MacDaniels

(415) $431-3800$

Fourp1y, Inc.

P.O. Box 890

Grants Pass, OR 97526

Maurice Saunders

(503) 479-3301

Guaranty Performance Company, Inc.

P.0. Box 748

Independence, KS 67301

Andrew Livingston

(316) 331-0027

Oregon State University

Department of Forest Products

Corval11s, OR 97331

Raymond A. Currier

(503) $753-9166$ 
1. Densification (Concluded)

Papakube Corporation

Energy Cube Division

931 East Harbor Drive

San Diego, CA 92101

Jerry Nelson

(714) 231-1490

Sprout Waldron Company, Inc.

Metal Products Division

Muncy, PA 17756

Kenneth Sternett

(717) $546-8211$ 
D. Conversion Technologies

2. Thermochemical Processes

a. Direct Combustion*

Abitibi Paper Company

Mississauga

Ontario LSK 1 A9 Canada

F. L. Morton

(416) 822-4770

Subject: Paper Plant Waste Burning

Bowater Southern Paper Company

Mill Engineering nepartment.

Calhoun, TN 37309

S. E. Bettis

(615) 336-2211

Subject; Burning of Wood Residues to Supply Some Mill Energy

Requirements

Burlington Electric Company

Burlington, VT 05401

(802) 658-0300

Subject: 50Mw Wood-Fired Power Plant

Under Construction

Energex, Ltd.

P.0. Box 4208

North Portland, OR 97208

Lloyd Day

(503) 286-8231

Subject: Commercialization of the

Jasper-Kock Suspension Burner

for Wet Woody Fuels

* For an additional listing of companies involved in direct combustion technology, refer to section II on Wood Boiler Firms 
a. Direct Combustion (Continued)

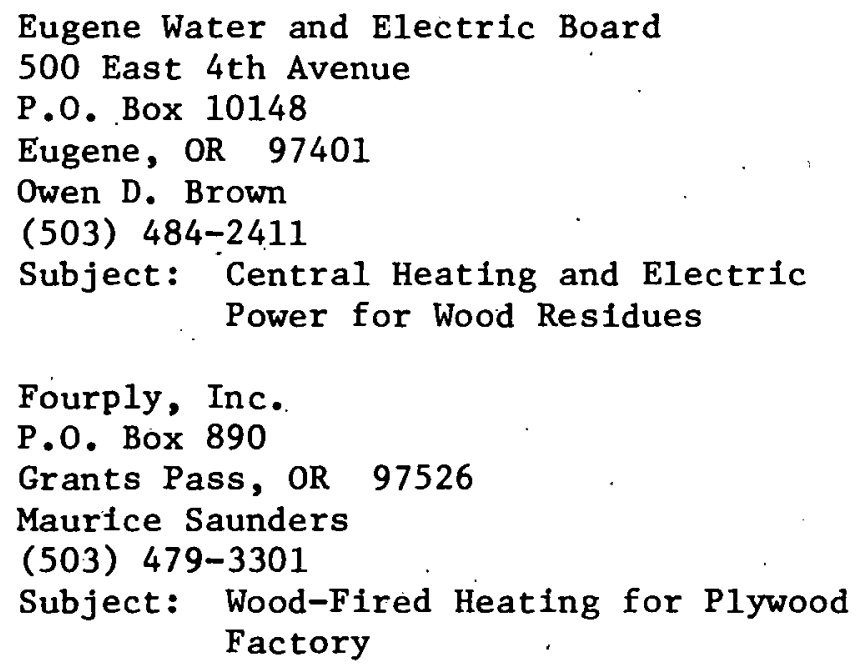


a. Direct Combustion (Continued)

Moore-Oregon-Canada

P.0. Box 4208

Portland, OR 97208

(503) 286-8231

Nor'west-Pacific Corporation

Suite 600, P1aza 600 Building

Seattle, WA 98101

Donald $W$. Pingrey

(202) 623-7224

Subject: Analysis of Wood-fired

Electric Power Generating

P1 ants

Sonoco Products

North 2nd Street

Hartsville, SC 29550

J. L. Dieh1

(803) 383-7255

Subject: Conversion of Bark and Other Residues into Steam for

Electric Generacion

Tennessee Valley Authority

Norris, TN 37828

Larry Klein

(615) 546-6912

Subject: Wood as`Fue1

U1trasystems, Inc.

2400 Michelsun Drive

Irvine, CA 92715

(714) $752-7500$

Washington Office:

Robert Kenne1

(703) 821-1960

Subject: Wood Furnace Development

University of Maine

Department of Agricultural Engineering Orono, ME 04473

John G. Riley

Subfect: Develop Automated System for

Heating Large Buildings with

Forest Res1dues as Fuel 
a. Direct Combustion (Concluded)

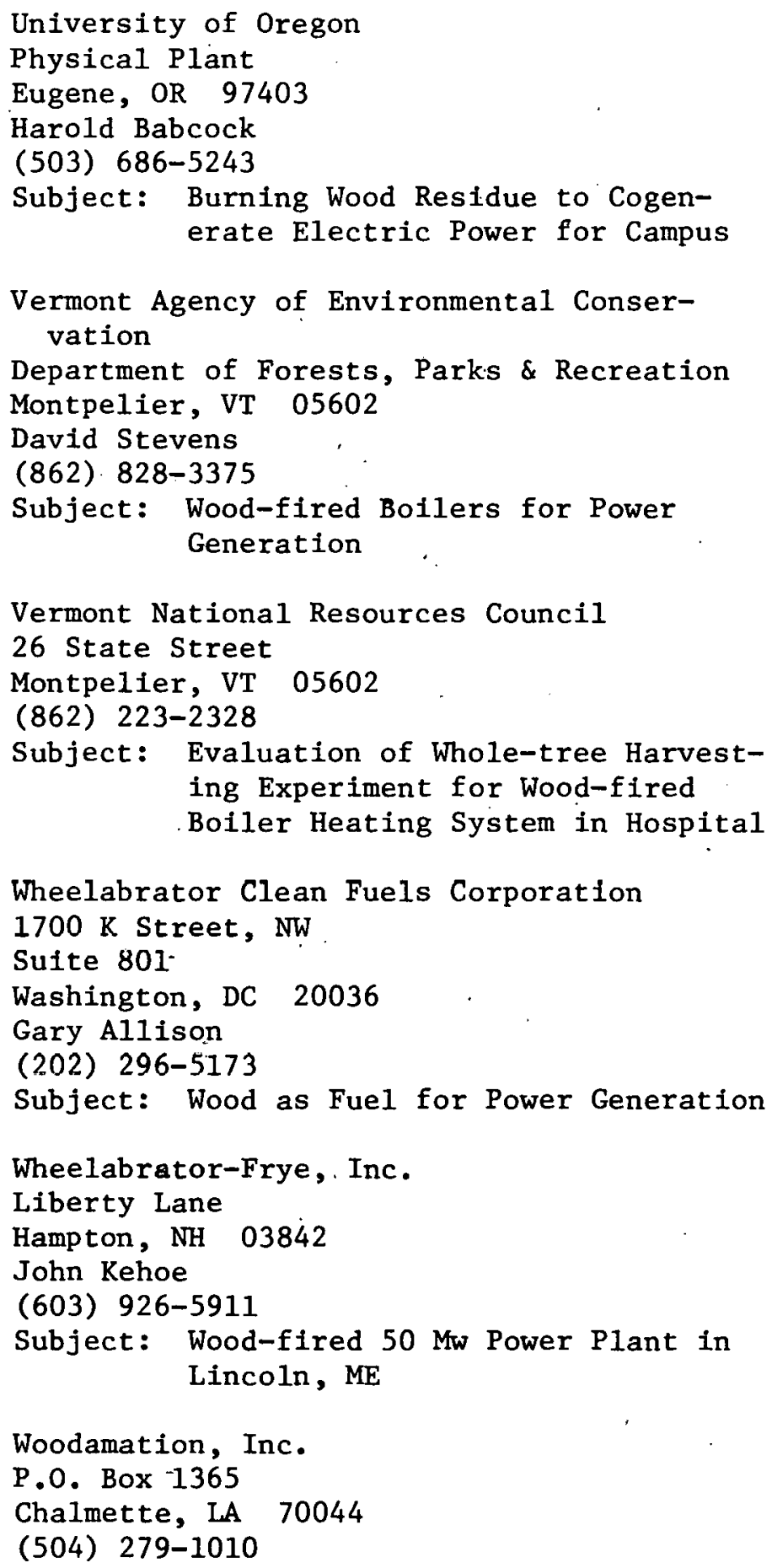




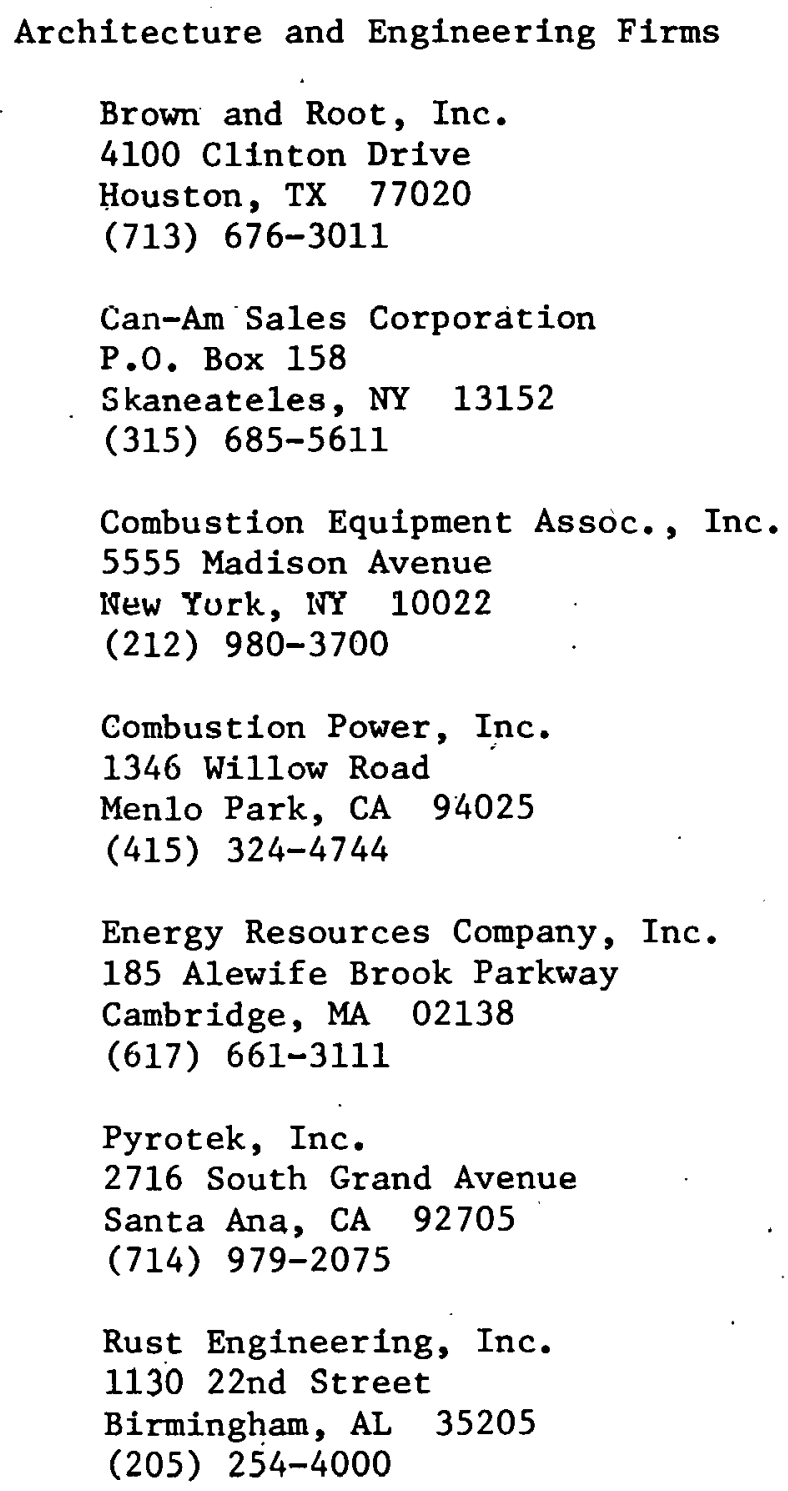


b. Gasification/Pyrolysis.

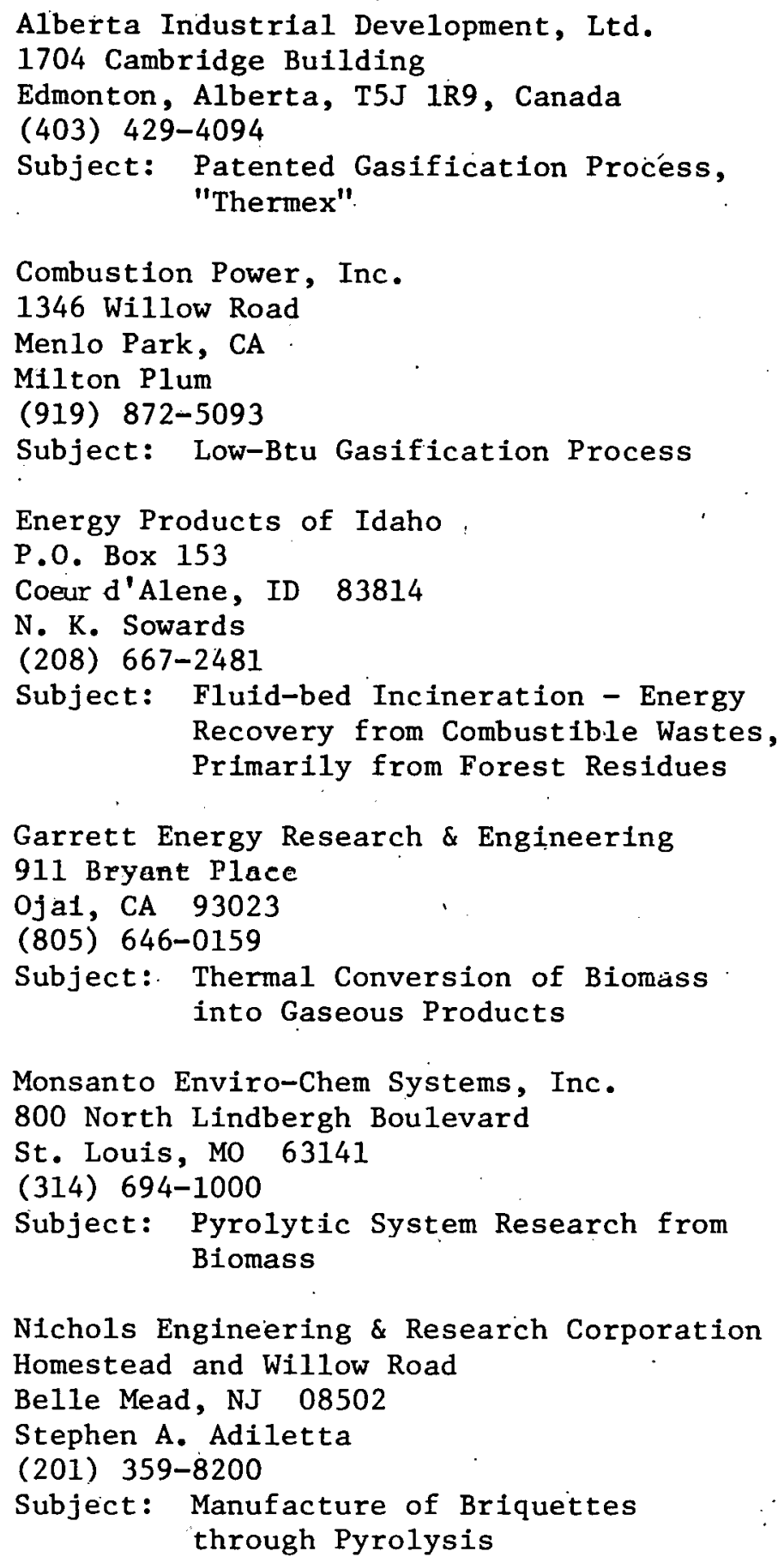


b. Gasification/Pyrolysis (Continued)

Occidental Research and Development Company 10889 Wilshire Boulevard

Los Angeles, CA 90024

(213) 879-1700

Subject: Pyrolysis Gas from Biomass

Pioneer.Hi-Bred International, Inc. 5700 Merle Hay Road

Johnson, IA 50131

Walter E. Stohlgren

(515) 245-3721

Subject: Corn Cob Gasifier

TcehAir Corpuraliun

2231 Perimeter Park

Atlanta, GA 30341

P. F. VanKeuren

(404) 458-9096

Subject: Pyrolysis of Wood

Texas Tech University

Lubbock, TX 79409

William J. Huf fman

(806) 742-3553

Subject: Synthesis Gas from Manure

Union Carbide Corporation

270 Park Avenue

New York, NY. 10017

(212) 551-2345

Subject: PUROX System for Wastes Utilization

University of West Virginia

Department of Chemical Engineering

Morgantown, WV 26506

Richard C. Bailie

(304) 293-0111.

Subject: Pyrolysis from Wood Residues

Weyerhaueser Company

Tacoma, WA 98401

William Munk

(206) 259-0425

Subject: Research on Wood Residues 
b. Gasification/Pyrolysis (Concluded)

Wright-Malta Corporation

Plains Road

Ballston Spa, NY 12020

J. A. Coffman

(518) 899-22.27

Subject: Design of Small Gasifier for

Biomass Residues 
c. Liquefaction/Pyrolysis

Battelle Memorial Institute

Pacific Northwest Laboratories

Battelle Boulevard

Richland, WA 99352

P. M. Molton

(509) 946-2121

Subject: Biomass to Liquids

Bechte1 Corporation

Scientific Development Department

50 Beale Street

San Francisco, CA 94119

$\mathrm{T}$. Lindemuth

(415) 768-1234

Subject: Wood-to-0i1

Exxon Corporation

1251 Avenue of the Americas

New York, NY 10020

(212) $398-3000$

General Motors Technical Center'

Warren, MI 48090

Robert L. Furey

(303) 575-2853

Subject: Ethanol Fuel Research

University of California

Lawrence Livermore Laboratory

Livermore, CA 94550

C. R. Wilke

(415) 422-1100

USDA/Forest Service

Forest Products Laboratory

Madison, WI 53705

Roger Rowell

(608) 257-2211

Subject: Liquid Chemicals from Woods 


\section{Bioconversion Processes}

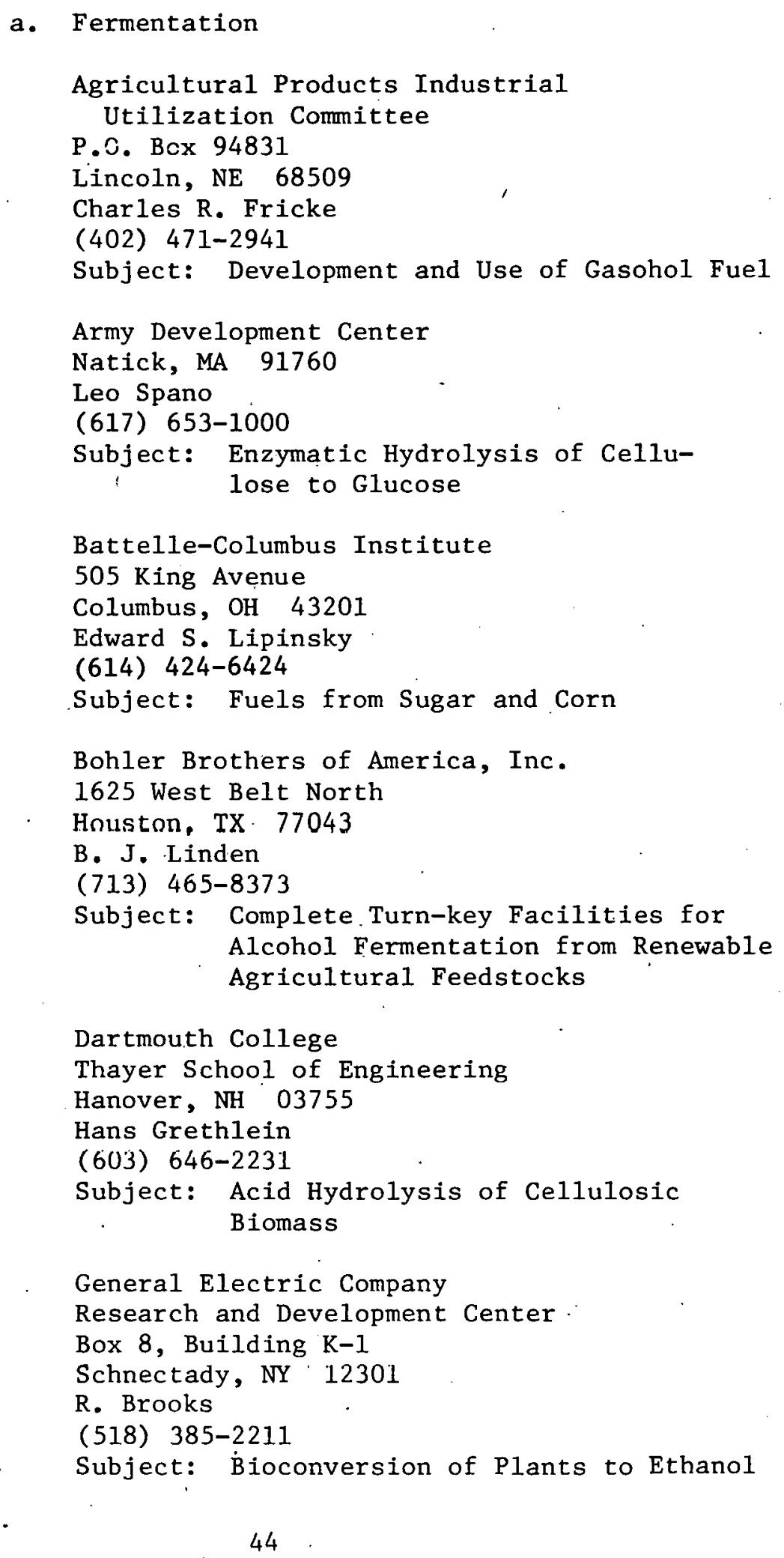


a. Fermentation (Continued)

Gulf Oil Chemicals Company

9009 West 67 th Street

Shawnee Mission

KS 66201

George H. Emert

(913) 722-3200

Subject: Commercialization of ethanol

from biomass by early 1980's

InterTech Group, Inc.

7768 Woodmont Avenue

Bethesda, MD 20014

Donald M. Browor

(301) 654-8762

Subject: Production of Ethanol from Carbohydrates in Algae Cultures

University of California

Lawrence Berkeley Laboratory

Berkeley, CA 94700

Charles A. Wilke

(451) 843-2740

Subject: Bioconversion of Cellulose to Ethanol.

Massachusetts Institute of Technology

Cambridge, MA 02139

Daniel I. C. Wang

(617) 253-2126

Subject: Fermentation of Suagrs from

Blomass

University of Nebraska

Department of Chemical Engineering

Lincoln, NE 68588

W. A. Scheller

(402) 472-7211

Subfect: Gasohol, an Alcohol Gasoline-

Additive from Grain

Purdue University

Renewable Resources Laboratory

West Lafayette, IN 47907

George Tsao

(31.7) 494-61.51

Subject: Fermentation Using Cellulosic Wastes 
a. Fermentation (Concluded)

Solar energy Research Institute 1536 Cole Boulevard

Golden, CO 80401

Thomas Reed

(303) 234-7127

Subject: Alcohol and Gasohol Fuels

Rensselaer Polytechnic Institute

Department of Chemical Engineering

Troy, NY 12181

Henry Bungay

(518) 270-6369

Subject: Fermentation of Biomass

Rutgers University

Cook College

New Brunswick, NJ 08900

Douglas E. Eveleigh

(201) 932-1766

University of Pennsylvania

College of Engineering and Applied Science

Philadelphia, PA 19174

E. K. Pye

(2i5) $243-7244$

Subject: Biological Production of Organic Solvents from Cellulosic Wastes

University of Toronto

Department of Chemical Engineering

Toronto M5S 1A4 Canada

Morris Wayman

Subject: Ethanol for Motor Fuel from

Biomass 
b. Anaerobic Digestion

Bio-Gas of Colorado, Inc.

American National Bank Building

Denver, CO 80202

Will lam Pace

(303) 572-1061

Subject: Bloconversion of Agricultural

Residues

Cornell University

Department of Agricultura1 Engineering

Ithaca, NY 14853

W. J. Jewe 11

(607) 256-4533

Subject: Potential of Methane Generation in Agriculture

Dynatech Research and Development Company 99 Erie Street

Cambridge, MA 02139

(617) 868-8050

Subject: Engineering Evaluation of Programs to Recover Fuel Gas from Waste

Ecotope Group

2332 East Madison

Seattle, WA 98112

Kenneth Smith

(206) $322-3753$

Subject: Digester Applications for Dairy Farm Operatinns

Georgia Institute of Technology

Engineering Experiment Station

Atlanta, GA 30332

(404) 894-3095

Subject: Anaerobic Digestion of Poultry Manures

Hamilton Standard

Windsor Locks, CT 06096

Robert E. Breeding

(203) 623-1621

Subject: Pipeline Fuel Gas from Experimentai Cattle Feedlot 
b. Anaerobic Digestion (Continued)

Pacific Gas \& Electric Company

245 Market Street

San Francisco, CA 94106

Pablo Macie1

(415) 781-4211

Southern California Gas Company

810 S. Flower Street

Los Angeles, CA 90017

Vince Bendanillo

(213) 689-3421

Subject: Jointly Sponsored Effort by Above Utilities to Investigate Feasibility of SNG Production from Cattle Manure

Sheaffer \& Roland, Inc.

20 North Wacker Drive

Chicago, IL . 60606

John Martin

(312) 236-9106

Subject: Anaerobic Digesters from

Cattle Waste

Stanford University

Dopartment of Civil Engineering

Palo Alto, CA 94305

Perry L. McCarty

(415) 497-3504

Subject: Methane Production

University of California

Agricultural Engineering Department

Davis, CA 95616

D. J. Hills

(916) 752-7420

Subject: Methane Generation from Crop

and Food Processing Residues

University of Illinois

Civil Engineering Department

Urbana, IL 61801

J. T. Pfeffer

(217) 333-6965

- Subject: Biological Conversion of

Blomass to Methane 
b. Anaerobic Digestion (Continued)

University of Minnesota

Department of Agricultural Engineering

St. Paul, MN 55108

(612) 373-0764

Phillip Goodrich

Subject: Feasibility Study of Blo-gas

Powered Electric Generator

\section{USDA/AES}

229 Agricultural Administration Building

Pennsylvania State University

University Park, PA 16802

J. M. Beattie

(814) 865-25/1

Subject: Methane from Manure

USDA/AES

Coastal Plain Station

Tifton, GA 31794

E. B. Browne

(912) 386-3338

Subject: Farm Manures

USDA/AES

College of Agricultural \& Environmental

Sciences

University of California

Davis, CA 95616

C. E. Hess

(916) 752-1605

Subject: Manure Conversion Study

USDA/AES

Colorado State University

Fort Collins, r.0 80523

J. P. Jordan

(303) 491-5371.

Subject: Manure Conversion Study 
b. Anaerobic Digestion (Concluded)

USDA/AES

College of Tropical Agriculture

University of Hawaii

2545 The Mall

Honolulu, Hawaii 96822

W. R. Furtick

(808) 948-8234

Subject: Bfoconversion of Organic Wastes

\section{USDA/AES}

University of I11inois

Urbana, IL 61801

G. W. Salisbury

(217) 333-0240

Subject: Methane Production

USDA/ARS

Northern Regional Research Center

1815 North University

Peoria, IL 61604

Dwight Miller

(309) 685-4011

Subject: Methane from Manure

IISDA / ARS

University of Missouri

Columbia, MO 65201

James R. Fischer

(314) 882-8337

Subject: Develop Methods of Equipment

for On-farm Anaerobic Digestion

of Agricultural Research

USDA/ARS

P. 0. Box 166

Clay Center, NE 68933

G. Hashimoto

(402) 762-3241

Subject: Anaerobic Digestion of Livestock and Crop Residue 


\section{RECIPIENTS OF INFORMATION}

\section{A. Federal Agencies*}

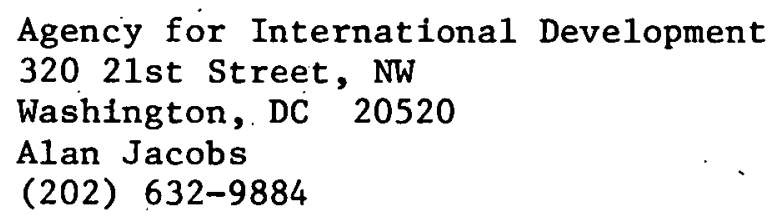

*Most Federal agencies are also good sources of information in specialized areas of biomass development. 
A. Federal Agencies (Continued)

Energy, Department of Division of Transportation Energy Conservation Washington, DC 20545

Eugene Ecklund, Chief

Alternative Fuels Utilization Branch

Energy, Department of

Office of Public Affairs

Washington, DC 20585

Jim Bishop, Director

(202) 376-5729

Environmental Protection Agency

401 M S treet, NW

Washington, DC $\cdot 20460$

Kurt Jacobson

(202) 755-9014

Environmental Quality, Council on

722 Jackson Place,' NW

Washington, DC 20006

John Davidson

Staff Member for Energy Programs

(202) 633-7097

National Academy of Sciences

2101 Constitution Avenue, NW

Washington, DC 20418

Philip Ross

(202) $389-6116$

National Science Foundation

Advanced Energy \& Resources Technology

1800 G Street, NW

Washington, DC 20007

Oskar Zaborsky

(202) 632-7398

State, Department of

International Energy R\&D

IESNETIM

Washington, DC 20540

Martin Prochnik

(202) 632-4413 
A. Federal Agencies (Concluded)

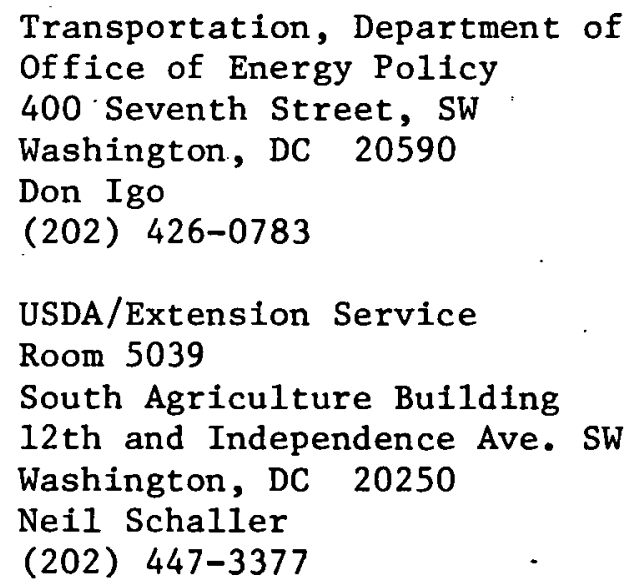

Agriculture, Department of Secretary's Office Administration Building Room 226-E

Washington, D.C. 20250

Weldon Barton, Director of Energy (202) 447-2455 
B. State Agencies

Energy Offices

Alabama, State of

Alabama Energy Board Management

Alabama Development office

State Capitol

Montgomery, AL 36130

Edwin G. Hudspeth

(205) 832-5010

Alaska, State of

Alaska Energy Office

Division of Fnergy \& Pnwer Development

Department of Commerce \& Economic

Development

7th Floor, McKay Bullding

Anchorage, AK 99501

William C. McConkey

(907) 272-0527

American Samoa

Government of American Samoa

Pago Pago, A.S. 96799

Ray Coston

Special Assistant to the Governor

9-976-1221: ask for 633-4116

Arizona, State of

Energy Program Section

Capitol Tower, Room 507

Plıveinix, AZ 85007

James Warnock

(602) 271-5371

Arkansas, State of

960 Plaza West

Little Rock, AR 72205

Mack B. Woodward

State Energy Coordinator

(501) 371-1374

California, State of

Energy Resources Conservation \& Development Commission

1111 Howe Avenue

Sacramento, CA 95825

Richard M. Maullin

(916) $322-3690$ 
B. State Agencles (Continued)

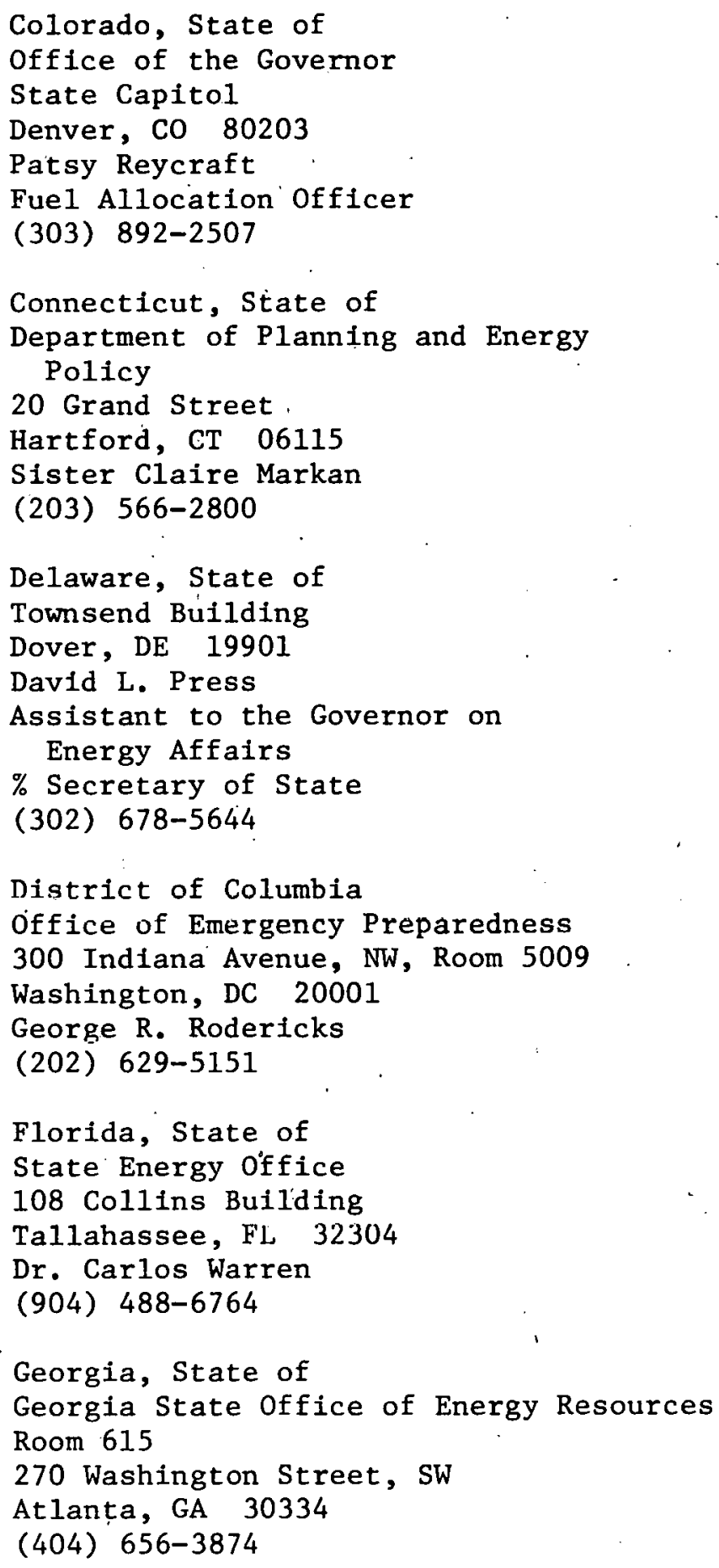


B. State Agencies (Continued)

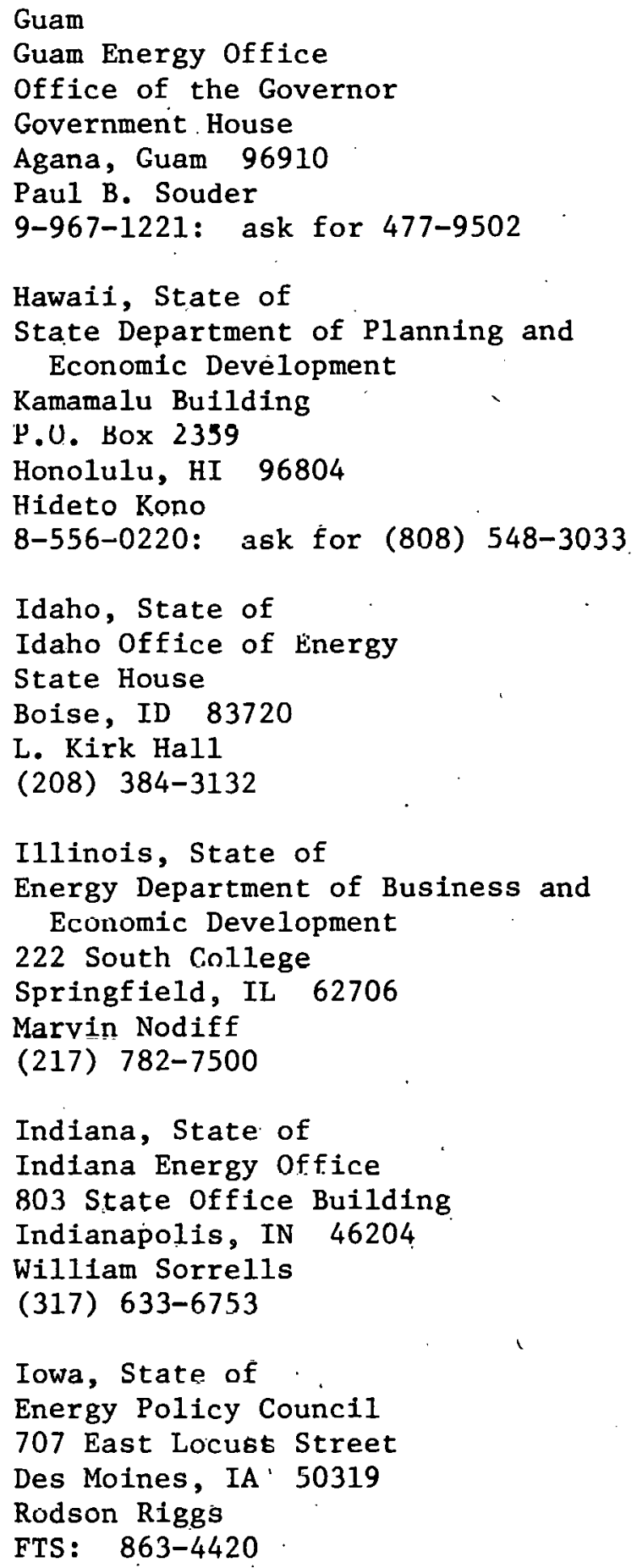




\section{B. State Agencies (Continued) -}

Kansas, State of State Energy Office

503 Kansas Avenue, Room 241

Topeka, KS 66603

Stephen D. Harris

(913) 296-2496

Kentucky, State of

Kentucky Department of Energy

Capitol Plaza Towers

Frankfort, KY 40601

Damon W. Harrison

(502) 564-7416

Louisiana, State of

Department of Conservation

P.0. Box 44275

Baton Rouge, LA 70804

Raymond J. Sutton

(504) 389-5161

Maine, State of

Office of Energy Resources

55 Capitol Street

Augusta, MF $n 4330$

Gary R. Linton

(207) 289-2196

Maryland, State of

Energy Policy office

301 West Preston Street

Suite 1302

Baltimore, MD 21201

John P. Hewitt

(301) 383-6810

Massachusetts, State of

Massachusetts Energy Policy Office

John W. McCormick Building

Room 1413

1 Ashburton Place

Boston, MA 02108

Henry Lee

(617) $727-4732$ 


\section{B. State Agencies (Continued)}

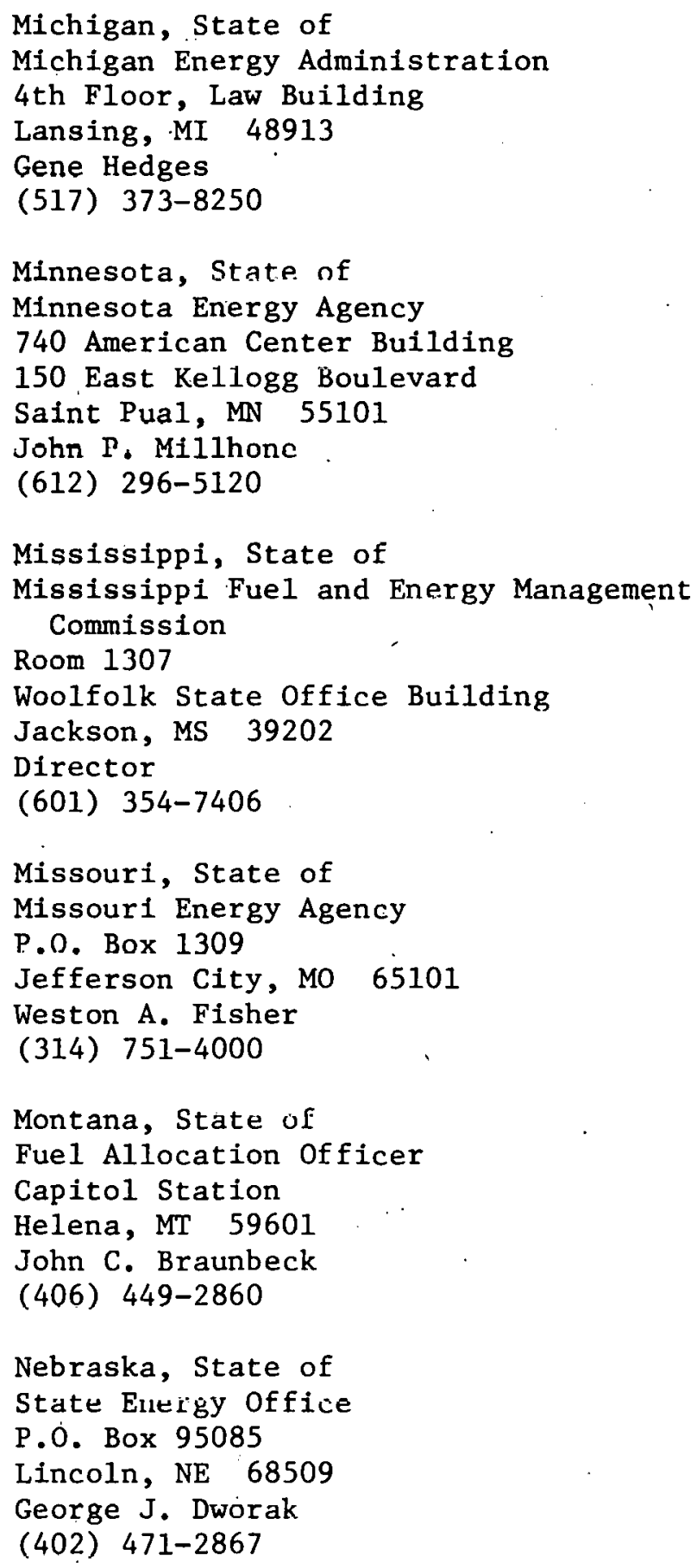




\section{B. State Agencies (Continued) \\ Nevada, State of \\ Public Service Commission \\ 505 East King Street \\ Carson City, NV 89710 \\ Noel Clark \\ (702) $885-4180$}

New Hampshire, State of

Governor's Council on Energy

3 Capitol Street

Concord, NH 03301.

Marshall Cobleigh

(603) 271-2121

New Jersey, State of

Department of Energy

101 Commerce Street

Newark, NJ 07102

(201) $648-3290$

New Mexico, State of

Energy Resources Board

P.O. Box 2770

Santa Fe, NM 87501

Fred $0^{\prime}$ Cheskey

(505) 827-2472

New York, State of

State Energy Office

Empire State Plaza

Swan Street Building

Core 1, Floor 2

Albany, NY 12223

James L. Larocca

(518) $474-2870$

North Carolina, State of

Energy Division

215 East Lane Street

Raleigh, NC 27611

Brian F1attery

(919) 733-2230 


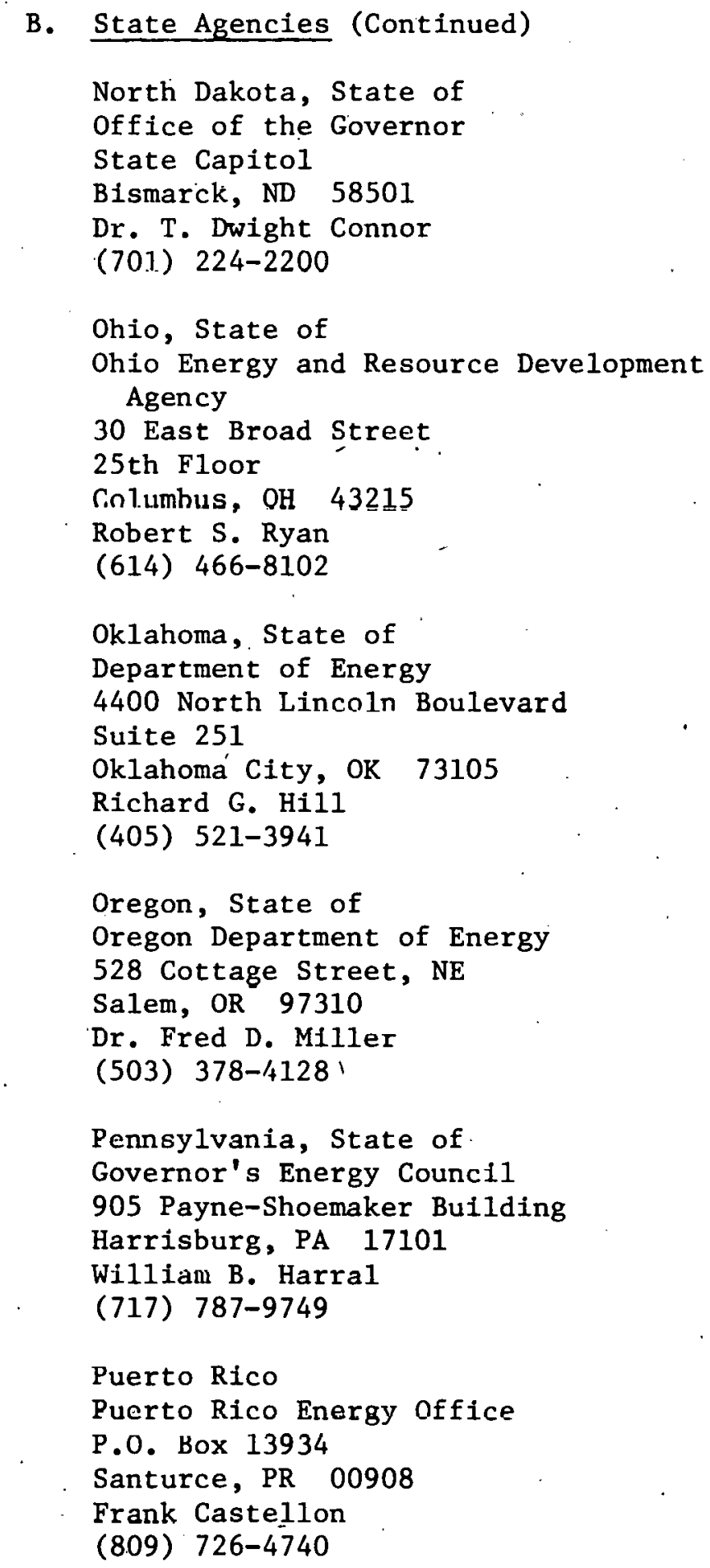




\section{B. State Agencies (Continued)}

Rhode Island, State of

State Energy Office

State House

Providence, RI 02903

Dante Ionata

(401) $277-3370$

South Carolina, State of

Energy Management Office

Edgar A. Brown Office Building

1205 Pendleton Street

Columbia, SC 29201

Steve Connor

(803) 758-2050

South Dakota, State of

Energy Policy Office

Joe Foss Building

Pierre, SD 57501

James A. Van Loan

(605) 224-3603

Tennessee, State of

Tennessee Energy office

2.50 rapital Hill Bullding

Nashville, TN 37219

Edward J. Spitzer

(615) 741-2994

Texas, State of

7703 North Lamar

Austin, TX 78752

Alvin E. Askew

Assistant to the Governor for Energy

Resources

(512) 475-5491

Utah, State of

Department of Natural Resources

438 State Capitol

Salt Lake City, UT 84114

(801) 533-5356 
B. State Agencies (Continued)

Vermont, State of

State Energy Office

State Office Building

Montpelier, VT 05602

Brendan Whittaker

(802) 828-2768

Virginia, State of

Virginia Energy Office

823 East Main Street

Room 300

Richmond, VA 23219

Louis R. Lawson, Jr.

(804) 786-8451

Virgin Islands

Virgin Islands Energy Office

Office of Budget Director

P.0. Box 90

Saint Thomas, VI 00801

Bruce Potter

(809) 774-0750

Washington, State of

Washington Energy Office

1000 South Cherry Street

Olympia, WA 98504

Lawrence B. Bradley

(206) 753-2417

West Virginia, State of

Fuel and Energy Office

$1262 \frac{1}{2}$ Greenbrier Street

Charleston, WV 25311

John D. Anderson III

(304) $348-8860$

Wisconsin, State of

Office of State Pianning and Energy

Room B130

1 West Wilson Street

Madison, WI 53702

Vicki Potter

(608) 266-3382 
B. State Agencies (Concluded)

Wyoming; State of

Department of Economic Planning and

Development

Barrett Building

Cheyenne, WY 82002

John P. Goodier

(307) $777-7284$ 


\section{Trade Associations*}

American Association of Cereal Chemists, Inc. 8340. Pilot Knob Road

St. Paul, MN 55121

Raymond J. Tarleton

(612) $454-7250$

American Dairy Association

6300 North River Road

Rosemont, IL 60018

Alden G. Grimes

(312) 696-1880

Amer lcall Papel Inslitite, Inc.

260 Madison Avenue

New York, NY 10016

Jeffrey Duke

(212) 883-8000

American Pulpwood Association

1619 Massachusetts Avenue, NW

Washington, DC 20036

(202) 265-0670

Canada Grains Counc11

400-177 Lombard Avenue

Winnipeg, Manitoba

Canada R3B OW5

Donald A: Dever

(204) 942-2254

Council of State Chambers of Commerce

Room 1018

1028 Connecticut Avenue, NW

Washington, DC 20036

(202) 296-2630

Farmer's Educational \& Cooperative Union of America

12025 East 45th Avenue

Denver, CO 80251

(303) $371-1760$

*A complete listing of trade associations with additional Information may be had by consulting"The National Trade and Professional Associations of the United States and Canada and Labor Unions." 13th edition, 1978; Columbia Books, Inc., 734 15th St. NW, Washington, DC 20005. 
C. Trade Associations (Continued)

Forest Industries Council*

1619 Massachusetts Avenue, NW

Washington, DC 20036

George C: Cheek

(202) 667-7807

Forest Products Research Society

2801 Marsha11 Court,

Madison, WI 53705

Arthur B. Brauner

(608) 231-1361

Grain Sorghum Producers Association

1708-A 15th Street

Lubbock, TX 79401

Elbert Harp

(806) 763-4425

National Association of County Agricu1-

tural Agents

180 Milan Avenue

Norwalk, OH 44857

John K. We1ls

(419) 744-2334

National Association of Real Estate Brokers, Inc. 1025 Vermont Avenue, NW

Washington, DC 20005

Johnson T. McClurkin

(202) 638-7521

Nationa1 Association of Wheat Growers

1031 15th Street, NW.

Washington, DC 20005

Jerry Rees

(202) 466-8630

National Cattlemen's Association

Box 569

1001 Lincoln Street

Denver, CO 80201

George S. Spencer

(303) 861-1904

*Policy Making Federation of Nine Associations 
C. Trade Associations (Continued)

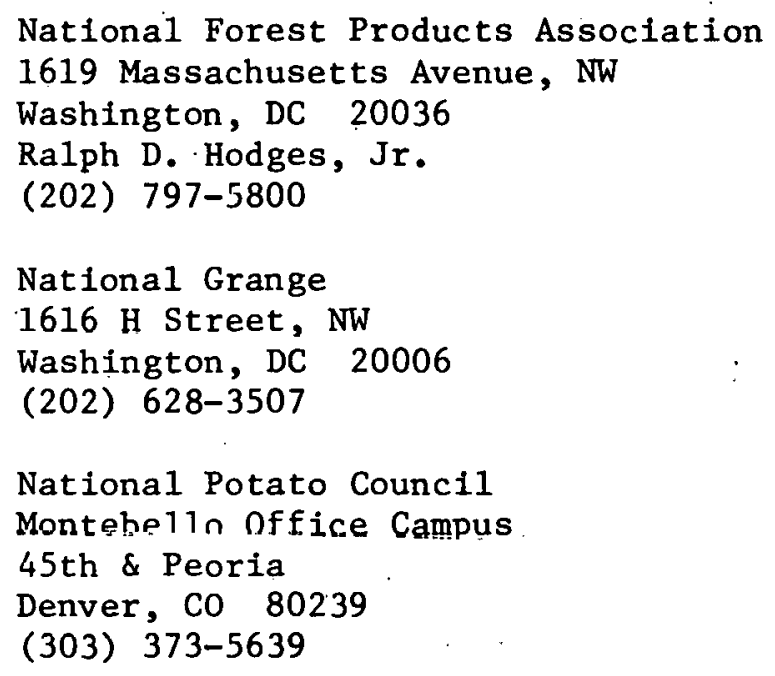




\section{Trade Associations (Continued)}

Pacific Logging Congress

217 American Bank Building

Portland, OR 97205

(503) 228-7971

Process Equipment Manufacturers Association P.O. Box 8745

Kansas City, MO 64114

(913) 642-3114

Pulp Chemicals Association

60 East 42nd Street

New York, NY 10017

(212) 697-4816

Pulp \& Paper Manufacutrers Association

1001 Connecticut Avenue, NW

Washington, DC 20036

(202) 785-4185.

Pulp, Paper \& Woodworkers of Canada

448 Seymour Street

Vancouver, British Columbia V6B $3 \mathrm{H} 3$

(604) 687-8591

Society of Wood Science and Technology

Box 5062

Madison, WI 53705

(608) 257-2211

Southern Forest Products Association

Box 52468

New Orleans, LA 70152

(504) 834-8544

Technical Association of the Pulp \& Paper Industry (TAPPI)

1 Dunwoody Park

At lanta, GA 30341

Herbert Teeple

(404) 394-6130 
C. Trade Assoclations (Concluded)

Timber Products Manufacturers

951 East Third Ayenue

Spokane, WA 99202

(509) 535-4646 
D. Utilities*

Edison Electric Institute

101518 th Street, NW

Washington, DC 20036

Glenn. H. Lovin

(202) 223-1265

Electric Power Research Institute

P.0. Box 10412

Palo Alto, CA 94303

(415) 855-2498

Gas Research Institute

$10 \mathrm{~W}$. 35th Street

Chicago, IL 60616

Ab Flowers.

(312) $567-6606$

Institute of Gas Technology

3424 S. State Street

IIT Center

Chicago, IL 60616

(312) $567-3650$

Southern California Edison Company

2244 Walmut Give

Rosemead, CA 91770

(213) 699-0211

United Gas and Pipeline Company

R\&D Department

P.0. Box 1478

Houston, TX 77001

Daniel Berkey

(713) 237-4123

\footnotetext{
*A number of utilities have projects in biomass conversion to electricity. Their names can be obtained by contacting the Electric Power Research Institute, Palo Alto, CA.
} 
E. Industry*

1. Forest Products

Black Clawson

P.0.' Box 1028

Everett, WA 98206

(206) 258-3555

Bowater, Incorporated

old Greenwich, CN 06870

Canadian International Paper

Sun Life Building

Mnnt.rea1, Quebec

Canada H3B 2XI

Champion International

One Landmark Square

Stamford, CN 06921

(203) $357-8500$

Container Corporation of America

1 First National Plaza

Chicago, IL 60670

(312) 786-5500

Crown Zellerbach Canada Ltd.

815 West Hastings Streets

Vancouver, Canada

(604) 668-4242

Crown Zellerbach Corporation

One Bush Street

San Francisco, CA 94119

Georgia-Pacific Corporation

900 South West 5th Avenue

Portland, OR 97204

*The following lists include a small sampling of industries which have expressed interest in bio-energy. Many of these industries are also engaged in biomass research, and could be considered sources of information on specialized aspects of bio-energy. 


\section{Forest Products (Continued) \\ Great Southern Paper Company \\ P.0. Box 44 . \\ Cedar Springs, GA 31732 \\ Hercules \\ 910 Market Street \\ Wilmington, DE \\ (302) 575-5000 \\ International Paper Company \\ 220 East 42nd Street \\ New York, NY 10017 \\ Koppers Company \\ Koppers Building \\ Pittsburg, PA 15219 \\ MacMillan Bloedel \\ 6540. Powers Ferry Road \\ Atlanta, GA 30339 \\ (404) 955-1324}

Masonite Corporation

29 . North Wacker Drive

Chicago, IL 60608

(312) $372-5642$

Morbark Industries

Winn, MI 48896

William E. Sells

(517) 866-2381

Olinkraft

P.0. Box 488

Jonesboro Highway

West Monroe, LA 71291

Owens-Corning Fiberglas Corporation

Fiberglas Tower

Toledo, OH 43659 
1. Forest Products (Concluded)

St. Regis Paper Company

150 East 42nd Street

New York, NY 10017

(212) $967-4400$

Scott Paper Company

Scott Plaza I

Philadelphia, PA 19113

(215) 521-5000

Timber Engineering Company 5530 Wisconsin Avenue Washington, DC 20015 (301) - 654-8288

Weyerhaueser Company

Tacoma, WA 98401

(206) $924-2345$ 


\section{Petrochemicals}

Allied Chemical Corporation

Columbia Road and Park Avenue

Morristown, NJ 07960

(201) 455-2000

Coyne Chemical Company

Comley Street and Delaware River

Philadelphia, PA 19135

(215) 831-8800

Dow Chemical Company

2030 Dow Center

Midland, MI 48640

(517) $636-1000$

E. I. DuPont de Nemours and Company

Central Research and Development Department

Wilmington, DE 19898

Robert M. Busche

(302) 774-1000

Monsanto Company

800 N. Lindbergh Boulevard

St. Louis, MO 63166

(314) 694-1000

Union Carbide Corporation

270 Park Avenue

New York, NY 10017

(212) 551-2345 


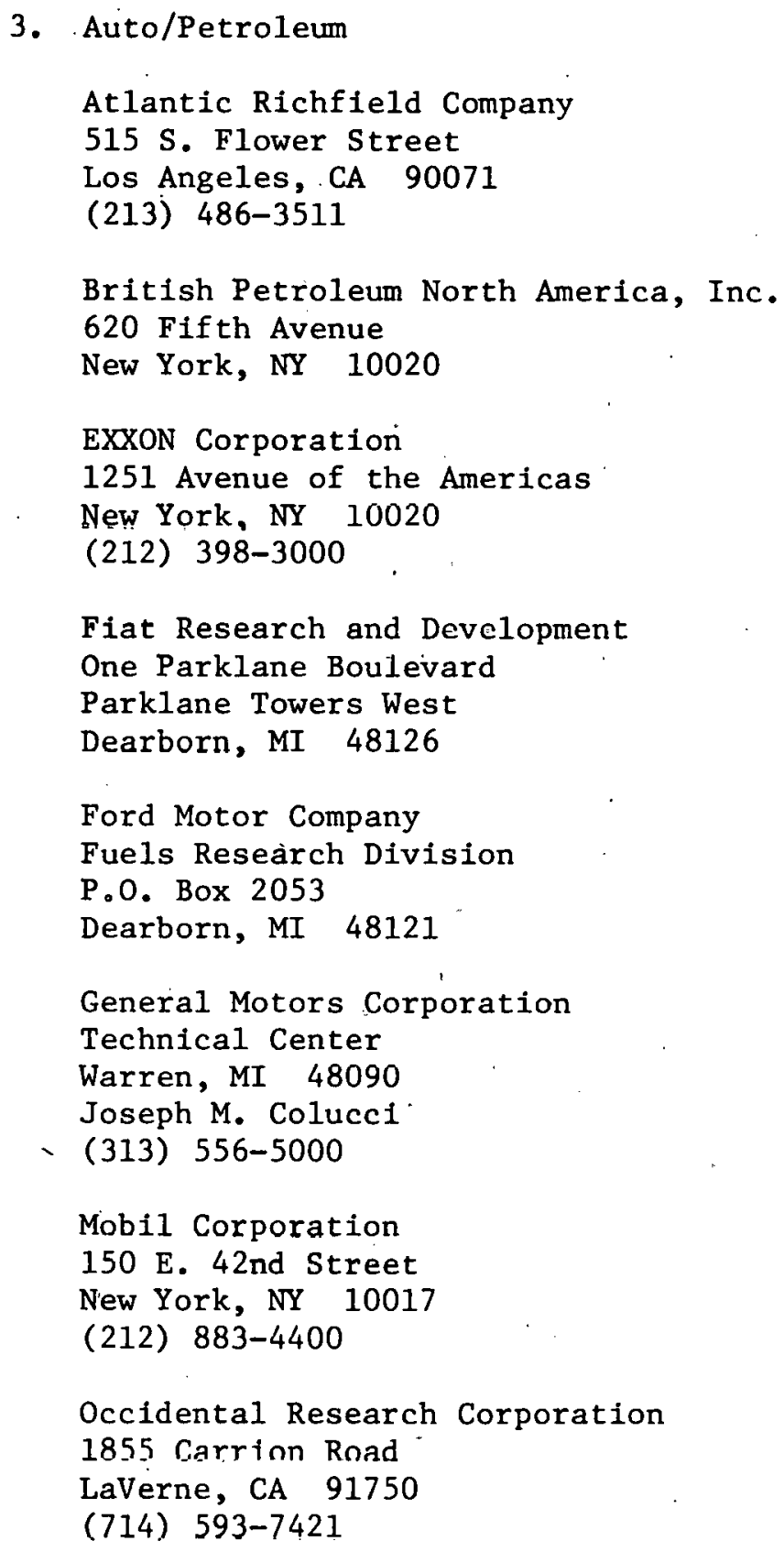


4. Wood Boiler Firms

American Fyr-Feeder Engineers

1265 Rand Road

Des Plaines, IL 60026

(312) 298-0044

Atlas Boiler and Equipment Company

W. 29 Spokane Falls Boulevard

Spokane, WA 99211

(503) 747-6001

Automated Combustion

P.O. Drawer 9

Lake Oswego, OR 97304

(503) 636-4569.

Babcock \& Wilcox Company

$20 \mathrm{~S}$. Van Buren Avenue

Barberton, OH 44203

(2i6) 753-4511

Bagot (Herman) and Company

3143 N. Nottingham

Chicago, IL 60634

(312) 637-6037

Basic Environmental Engineering, Inc.

21 H1ll Street

Glen Ellyn, IL 60137

(312) 469-5340

Bigeluw Company

P.0. Box 706

New Haven, CT 06503

(203) 772-3150

Bumstead Woolford Company

P.0. Box 448

Woodinville, WA 98072

(206) 485-9646

Burnham Corporation

P.0. Box 27

Lancaster, PA 17604

(717) $397-4701$ 


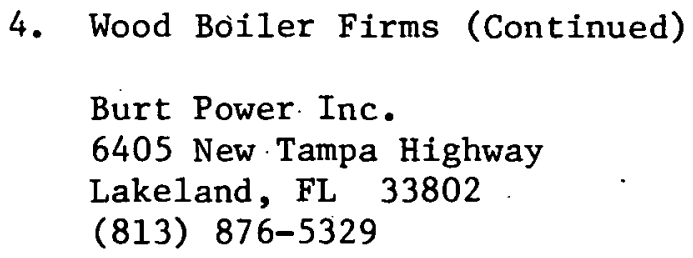

Combustion Engineering, Inc. 900 Long Ridge Road

Stamford, CT 06902

(203) 688-1911

Copeland Systems, Inc.

200 Spring Road, Suite 300

Oak. Brook, TT. Gก521

(312) 654-2820

Detroit Stoker Company

Manroe, MI 4816.1

(313) $241-9500$

Eclipse Lookout Company

P.0. Box 4756

Chattanooga, TN 37405

(615) 265-3441

Energex, Limited

P.0. Box 4208

North Portland, OR 97208

(503) 286-8231

Energy Control Engineering Corporation

P.0. Bux 3064

Charlotte, NC 28203

(704) 375-1701

Energy Products of Idaho

P.0. Box 153

Coeur d'Alene, ID 83818

(208) $667-6439$

Environmetrix

4725 University Way NE

Seattle, WA 98115

(206) $524-6350$ 
4. Wood Boiler Firms (Continued)

Foster Wheeler Energy Corporation

110 South Orange Avenue

Livingston, NJ 07039

(201) 533-1100

Gaskell Company, Inc.

P.O. Box 13225

Memphis, TN 38113

(901) 775-3222

Harvey Engineering \& Manufacturing Corp.

Route 2, Box 478

Hot Springs, AR 71901

(501) 262-1010

Industrial Boiler Company

221 Law Street

Thomasville, GA 31792

(912) 226-3024

International Boiler Company

E. Strousburg, PA 18301

(717) $421-5100$

Irvington-Moore Division of USNR

Y.U. Box 40666

Jacksonville, FL 32203

(509) 747-7965

Johnston Boiler Company

Ferrvburg, MI 49409

(616) 842-5050

Keeler (E.) Company

238 West Street

Williamsport, PA 17701

(717) 326-3361

Kewanee Boiler Corporation

101 North Franklin Street

Kewanee, IL 61443

(309) 853-3541 


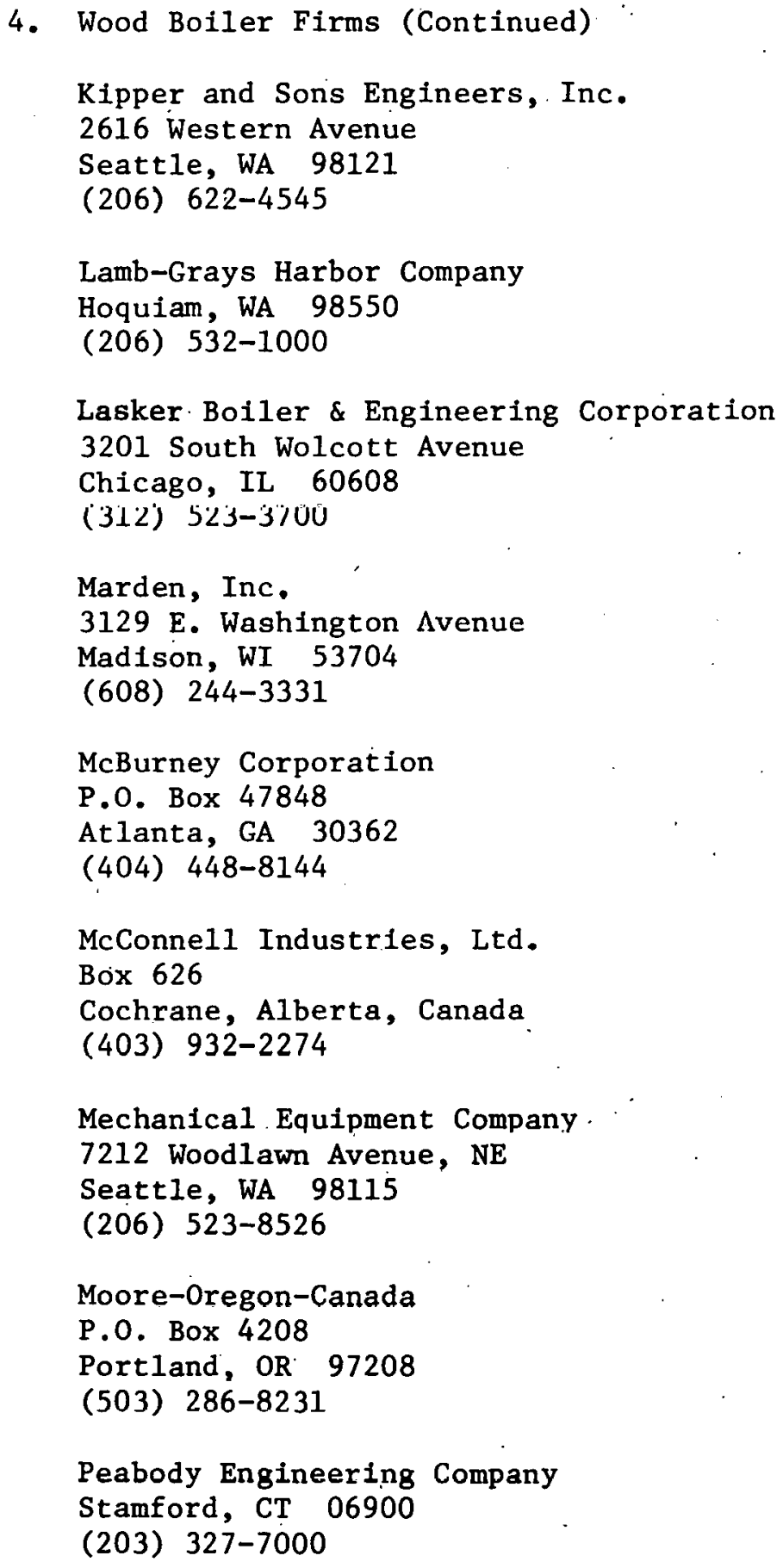


4. Wood Boiler Firms (Continued)

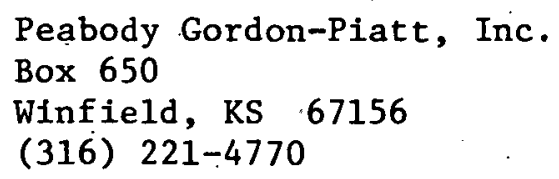

Pyrotechnic Industries, Limited

Box 626

Cochrane, Alberta, Canada

(403) 932-2274

Ray Burner Company

1303 San Jose Avenue

San Francisco, CA 94112

(415) 333-5800

Riley Stoker Company

9 Neponset Street

Worcester, MA 01613

(617) 852-7100

Rogers (John) Company

4605 Illinois Avenue

Louisville, KY 40213

(502) 458-5400

Seattle Boiler Company

5237 Marginal Way

Seattle, WA 98134

(206) 762-0737

Smith (Perry) Company, Inc.

P.O. Box 21282

Chat tanooga, TN 37421

(615) $982-7130$

Stearns-Roger, Inc.

P.O. Box 5888

Denver, CO 80217

(303) 758-1122

Steel Craft Corporation

Box 12408

Memphis, TN 38112

(901) 452-5200 
4. Wood Boiler Firms (Concluded)

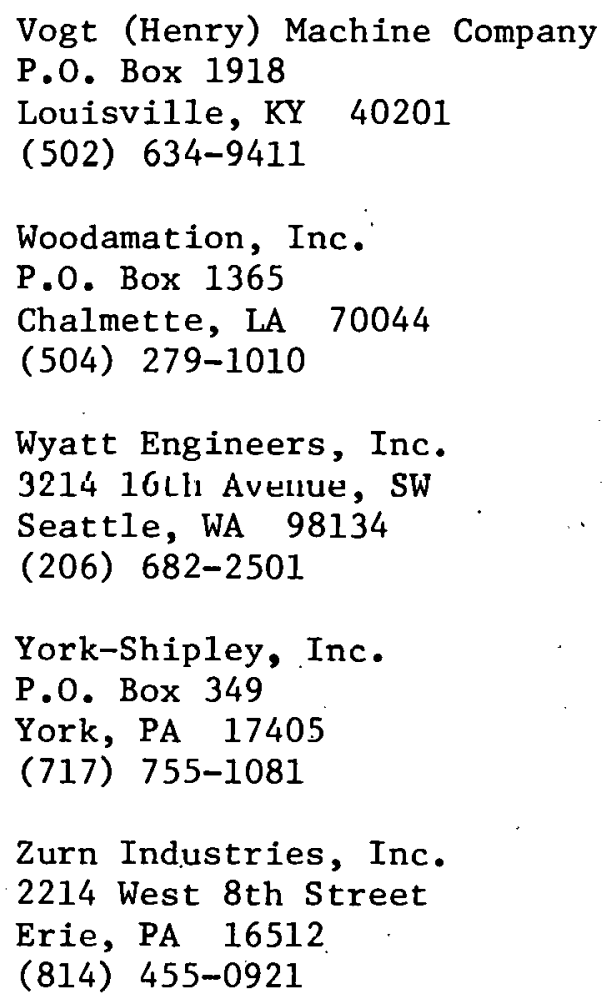


5. Other Industries*

J. P. Stevens

Box 2850

Greenville, SC 29602

National Realty Committee

2033 M Street, NW

Washington, DC 20036

Textile Workers Union of America AFL/CIO - Engineering Department

99 University Place

New York, NY 10003

Corning Glass Works

Sullivan Park

Corning, NY 14830

Grumman Ecosystems Corporation

1111 Stewart Avenue

Bethpage, NY 11714

Pratt and Whitney

Florida Research \& Development Center

West Palm Beach, FL 33402

IBM

1000/3000 Westchester Avenue

White Plains, NY 10604

United Technologies

P.0. Box 109

South Windsor, CN 06074

Leeds and Northrup Company

Dickerson Road

North Wales, PA 19454

American Can Cóporation

P.0. Box 50

Princeton; NJ 08540

*Listed here is a sampling of industries which have shown an interest in bio-energy development. 
F. Agriculture

1. Producers and Processors

Amstar Corporation

1251. Avenue of the Americas

New York, NY 10020

Arizona Feeds

P.0. Box 5526

Tuscon, AZ 85703

DeKalb Agricultural Research

Sycamore Road

DekaTh, TT. 6ח115

General Compost Corporation

322 South 16th Street

Philadelphia, PA 19102

General Mills Inc.

9200 Wayzata Boulevard

Minneapolis, MN 55440

Grace (W.R.) and Company

Agricultural Chemicals Group

P.0. Box 27147

Memphis, TN 38127

Kellog Company

235 Porter Street

Battle Creek, MI 49016

Nabisco Company

River Road \& DeForest Avenue

East Hanover, NJ 07936

Oscar Mayer and Company

Industrial Products and Processes Research

F.0. Box 1409

Mad1son, WI 53701

'l'he Pillsbury Company

401 Second Street, SE

Minneapolis, MN 55414 
1. Producers and Processors (Concluded)

Quaker Oats Company

Merchandise Mart Plaza

Chicago, IL 60654

Ralston Purina Company

835 S. Eighth Street

St. Louis, MO 63102 


\section{Machinery/Manufacturers \\ Allis-Chalmers Corporation \\ 1205 South 70th Street \\ Milwaukee, WI 53201 \\ Caterpillar Tractor Company \\ Marketing Department \\ 100 N.E. Adams Street \\ Peoria, IL 61629 \\ Deere and Company \\ John Deere Road \\ Moline, IL 61265 \\ International Harvester \\ 401 N. Michigan Avenue \\ Chicago, IL 60611}




\section{G. Alternative Energy Information Organizations*}

American Association for The Advancement of Science 1515 Massachusetts Avenue', NW Washington, DC 20036.

Allen Hammond (202) 467-4351

Conservation Foundation

- 1717 Massachusetts Avenue, NW Washington, DC 20036

Russel1 E. Train (202) 797-4370

Maine Audubon Society

Gilsland Farm

118 Route 1

Falmouth, ME 04105

(207) 781-2330

Nationa1 Wildlife Federation

1412 Sixteenth Street, NW

Washington, DC 20036

Thomas L. Kimbal1

(202) 797-6800

Overseas Development Council

1717 Massachusetts Avenue, NW

Washington, DC 20036

RAIN

$2270 \mathrm{NW}$ Irving Street

Portland, OR 97210

Lee Johnson

(503) 227-7225

Resources for the Future

1755 Massachusetts Avenue, NW

Washington, DC 20036

(202) 462-4400

The Rodale Press

Organic Park

Emmaus, PA 18049

(215) $967-9171$

*A comprehensive listing of organizations and officials concerned with natural resource use and management can be found in the Conservation Directory, 23rd Edition, published by the National Wildlife Federation. 
G. Alternative Energy Information Organizations (Concluded)

Sierra Club,

324 C Street, SE

Washington, DC 20003

Greg Thomas

(202) $547-1144$

Volunteers in Technical Assistance

3706 Rhode Is land Avenue

Mt. Rainier, MD 20822

(301) $277-7000$

Worldwatch Institute

1776 Massachusercs Avenue, INW

Washington, DC 20036

Dennis Hayes

(202) 452-1999 


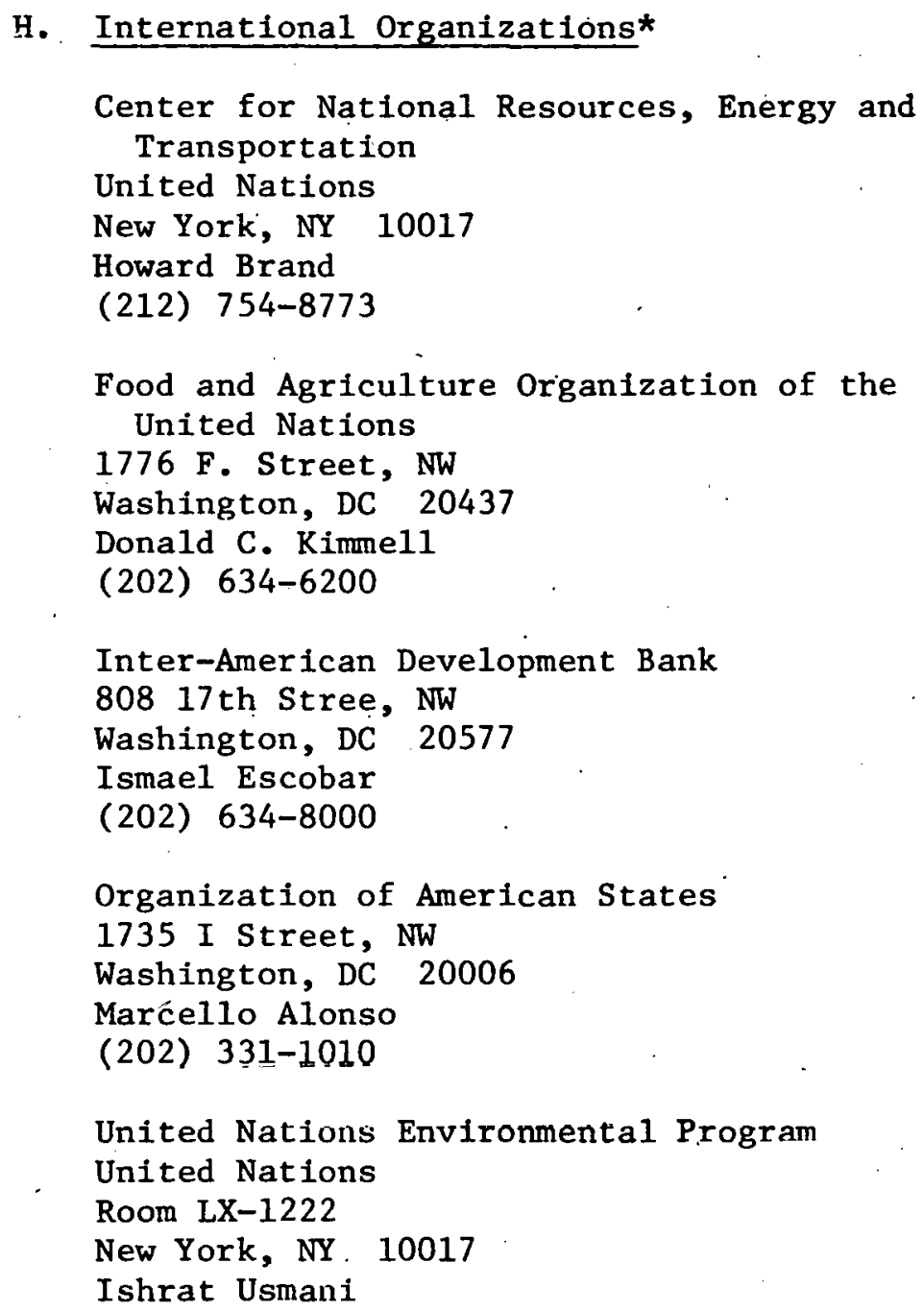
*Sclentific and/or Commercial attaches of all Embassies should be recipients.


Page

Abitibi Paper Company . . . . . . . . . . . . 35

Agency for International Development . . . . . . . . 52

Agnew Environmental Products ............... 33

Agricultural Products Industrial Utilization Comtittee . . 44

Alabama, State of - Alabama Energy Board Management . . . . 55

Alaska, State of - Alaska Energy Office . . . . . . . . 55

Alberta Industrial Development, Ltd. . . . . . . . . . 40

Allied Chemical Corporation . . . . . . . . . . . . 74

Allis-Chalmers Corporation ................ 85

American Association for the Advancement of Science . . . . 86

American Aooociation of Ccrcal Chemiat3, Ine. . . . . . . 65

American Can Corporation ............... 82

American Dairy Association . . . . . . . . . . . 65

American Fyr-Feeder Engineers . . . . .......... 76

American Paper Institute, Inc. . . . . . . . . . . . 65

American Pulpwood Association ............. 65

American Samoa ................... . . 55

Amstar Corporation .. . . . . . . . . . . . . 83

Arizona Feeds..................... 83

Arizona, State of - Energy Program Section ......... 55

Arkansas, State of - State Energy office . . . . . . . 55

Army Development Center............... . . . 44

Joseph E. Atchison, Inc. . . . . . . . . . . . . 13

Atlantic Richfield Company ............... 75

Atlas Boiler \& Equipment Company . . . . . . . . . 76

Automated Combustion ............... 76

Babcock \& Wilcox Company . . . . . . . . . . . . 76

Bagot (Herman) and Company . . ............ 76

Basic Environmental Englneering, Inc. . . . . . . . . 76

Battelle Memorial Institute (Colımbus). . . . . . . 13, 29, 44

Battelle Pacific Northwest Laboratories (Richland) . . . . 43

Bechtel Corporation . . . . . . . . . . . . . 43

Blgelow Company ................... 76

Bio-Energy Council, The . . . . . . . . . . . . . 12

Blo-Gas of Colorado, Inc. . . . . . . . . . . . . 47

Blomass Energy Institute, The ................ 12

Bio-Solar Research \& Develupment Curporation . . . . . . 33

Black Clawson . . . . . . . . . . . . . . . 71

Bohler Brothers of America, Inc. . . . . . . . . . . 44

Bonnot Company .. . . . . . . . . . . . . . . 33

Bowater, Incorporated ....... . . . . . . . . . 71

Bowater Southern Paper Company . . . . . . ... . . 35

Brltish Petroleum North America, Tnr. . . . . . . . . 75 
Brown and Root, Inc. . . . . . . . . . . 39

Bumstead Woolford Company . . . . . . . . . 76

Burlington Electric Company ............. . 35

Burnham Corporation .. . . . . . . . . . . . 76

Burt Power, Inc. . . . . . . . . . . . . . 77

California Institute of Technology . . . . . . . . 13, 30

California Pellet Mill Company ............. 33

California, State of - Energy Resources Conservation and . . 55

Development Commission

University of California ......19, 24, 28, 29, 43, 45, 48

Canada Grains Council ................ 65

Canadian International Paper . . . . . . . . . . 71

Can-Am Sales Corporation . . . . . . . . . . . . 39

Caterpillar Tractor Company . . . . . . . . . . . . 85

Champion International ................ 71

Colorado; State of - Office of the Governor . . . . . . 56

Columbia University . . . . . . . . . . . . . 30

Combustion Engineering, Inc. .. . . . . . . . . . . 77

Combustion Equipment Association, Inc. . . . . . . . 39

Combustion Power, Inc:................. 39, 40

Commerce, Department of - National Oceanic and Atmospheric . 52 Administration

Complete Tree Institute. . . . . . . . . . . . 16

Connecticut, State of - Department of Planning and Energy - 56 Policy

Conservation Foundation .. . . . . . . . . 86

Container Corporation of America . . . . . . . . . . 71

Copeland Systems, Inc. . . . . . . . . . . . . 77

Cornell University . . . . . . . . . . . . . . 47

Corning Glass Works . . . . . . . . . . . . . 82

Council of State Chambers of Commerce . . . . . . . . 65

Coyne Chemical Company . . . . . . . . . . . . . . 74

Crown Zellerbach Canada, Ltd. . . . . . ....... 71

Crown Zellerbach Corporation ............... 71

Dartmouth College ................... ... 13, 44

Deere and Company ................... 85

Defense, Department of - Energy office.......... 52

DeKalb Agricultural Research ................ 83

Delaware, State of - Assistant to the Governor on Energy - . 56 Affairs $r$

Detroit Stoker Company . . . . . . . . . . . 77

Development Planning \& Research Assoclates . . . . . . . 13

District of Columbia - Office of Emergency Preparedness . • 56 


\title{
INDEX (Continued)
}

\begin{abstract}
$\underline{\text { Page }}$
Dow Chemical Company'.................. . 74

E. I. DuPont Nemours and Company .... . . . . . . 74

Dynatech R\&D Company .............. 14, 30, 47
\end{abstract}

Eclipse Lookout Company . . . . . . . . . . . 77

Ecotope Group .. . . . . . . . . ...... . . 47

Edison Electric Institute . . . . . . . . . . 70

Electric Power Research Institute........... 70

Energex, Ltd. ................... 35, 77

Energy Control Englneering Corporation . . . . . . . . 77

Energy, Department of .................. 12, 53

Energy Products of Idaho . . . . . . . . . . . 4 40, 77

Energy Resources Company, Inc. . . . . . . . . . . . 39

Environmental Protection Agency . . . . . . . . . . . 53

Council on Environmental Quality . . . . . . . . . . 53

Environmetrix . . . . . . ............... 77

Eugene Water \& Electric Board . . . . . . . . . 36

Exxon Corporation ....................... 40, 43, 75

Farmer's Educationa1 \& Co-operative Union of America . . . 65

Fiat Research and Development . . . . . . . . . 75

Florida, State of - State Energy Office . . . . . . . 56

University of Florida . . . . ............ 19, 23

Ford Motor Company ... . . . . . . . . . . . 75

Forest Industrles Counc11 . . . . . . . . . . . 66

Forest Products Laboratory . . . . . . . . . . . . 17

Forest Products Research Society . . . . . . . . . . 66

School of Forest Resources and Conservation . . . . . . 2.1, 26

Foster Wheeler Energy Corporation' . . . . . . . . . 78

Fourply, Inc. ...................... 33, 36

Garrett Energy Research \& Engineering . . . . . . . . . 40

Gaskell Company, Inc. ................ 78

Gas Research Institute ............ . . : 30,70

General Compost Corporation , . . . . , . . . . : 83

General Electric Company ............... 31, 44

General Mills, Inc. . . . . . . . . . . . . 83

General Motors Corporation ............. 43, 75

Georgla Forestry Commission . . . . . . . . . . . 22

Georgia Institute of Technology .............. 21, 47

Georgla-Paciflc Corporation . . . . . . . . . . . 71

Georgia, State of - Georgia, State Office of Energy Resources. 56 


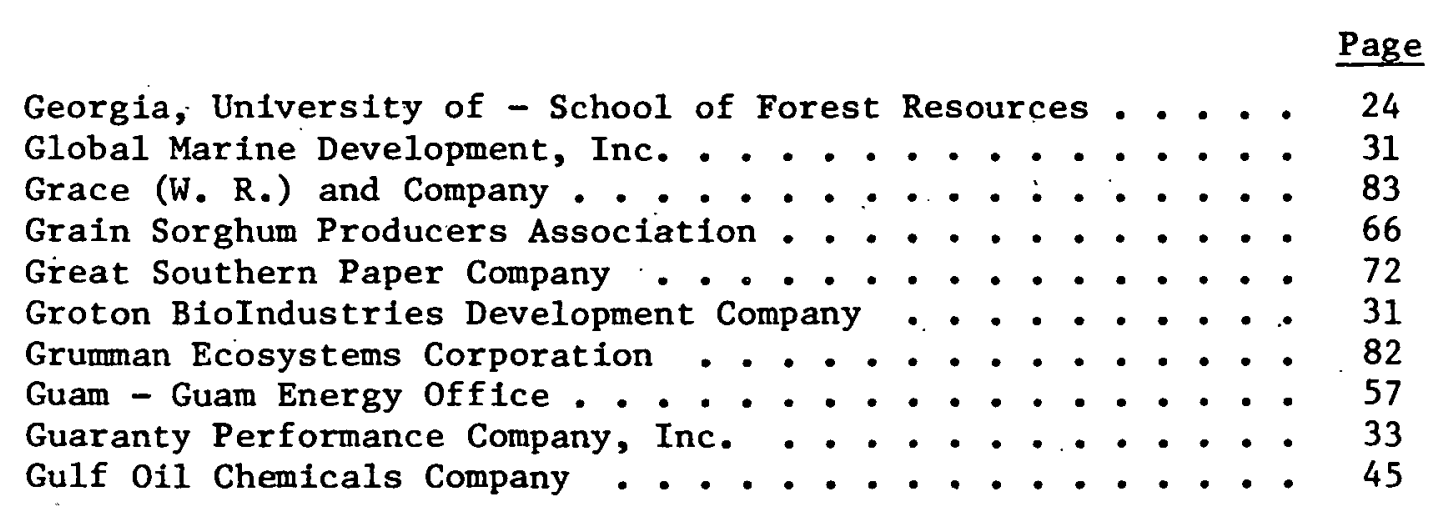

Hamilton Standard :............... 47

Harvey Engineering and Manufacturing Corporation ... . . 78

Hawaif, State of - State Department of Planning and Economic 57

Development

Hawailan Sugar Planters Assoclation . . . . . . . . 19

Hercules ..................... 72

Hilo Coast Processing Company . . . . . . . . . . 36

Houston, University of ................. 24

IBM ....................... 82

Idaho, State of - Idaho Office of Energy . . . . . . . 57

Illinois, State of - Energy Department of Business and . . 57

Economic Development

Illinois, University of - Civil Engineering Department 2.1, 48, 50

Indiana, State of - Indiana Energy Office . . . . . . . 57

Industrial Buller Company. ... . . . . . . . 78

Institute of Gas Technology .............. 14,70

Inter-American Development Bank ............. 88

International Boiler Company ............. . 78

International Harvester .. . . . . . . . . . 85

International Paper Company . . . . . . . . . . . 22, 72

InterTech Group, Inc. . ... . . . . . . . . . 45

InterTechnology/Solar Corporation . . . . . . . . . 14

Iowa, State of ................. . . 57

Iowa State University ................. . 21

Irvington-Moore Division of USNR ............. 78

Johnson Boiler Company . . . . . . . . . . . 78

Kansas, State of - State Energy Office . . . . . . . 58

Kansas State University . . . . . . . . . . . . 21

Keeler (E.) Company ..................... . 78

Kellog Company ....................... 83

Kentucky, State of - Kentucky Department of Energy . . . . 58

Kewanee Boiler Corporation . . . . . . . ... . 78

Kipper \& Sons Engineers, Inc. . . . . . . . . 36, 79

Koppers Company .................. 72 


\section{INDEX (Continued)}

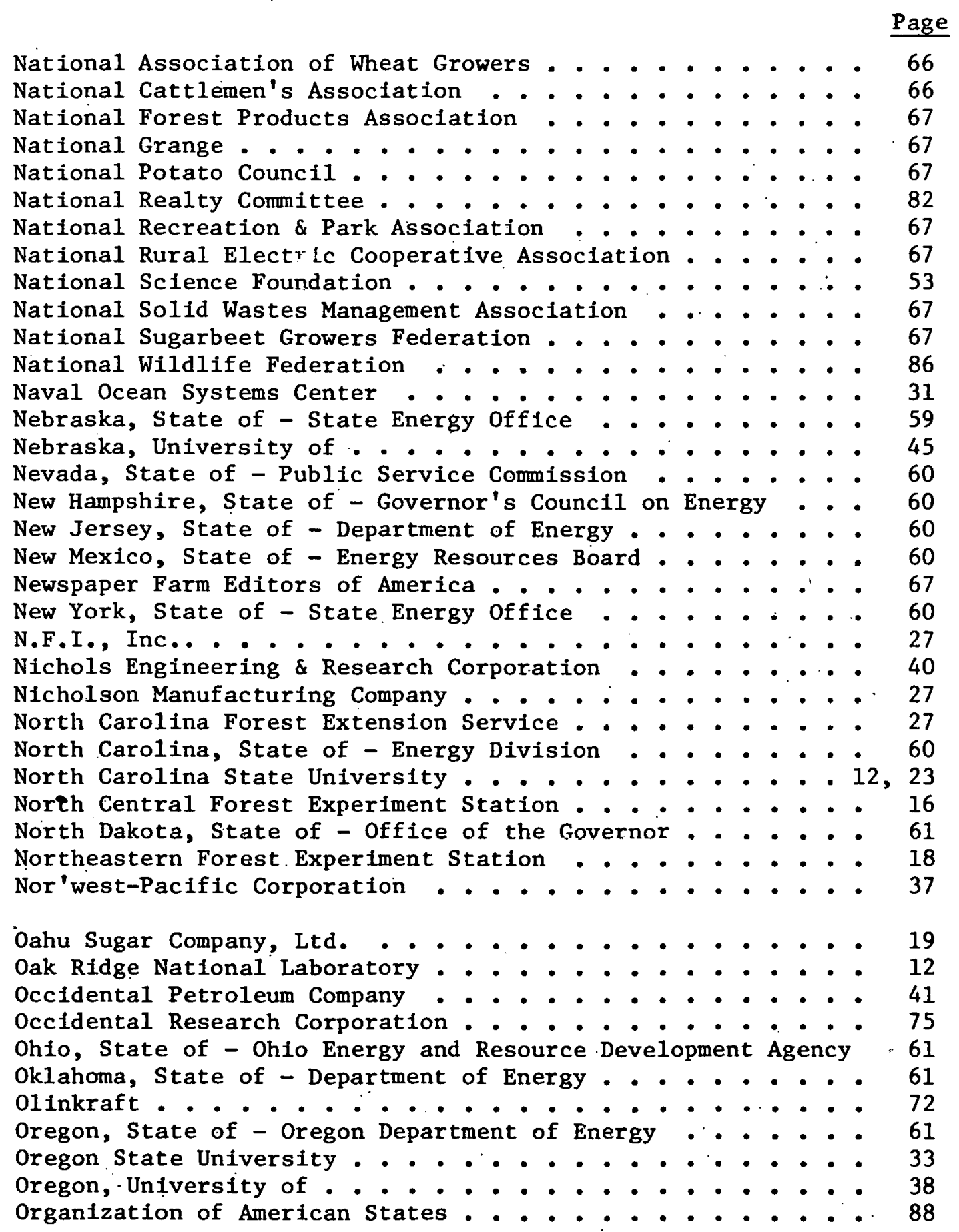




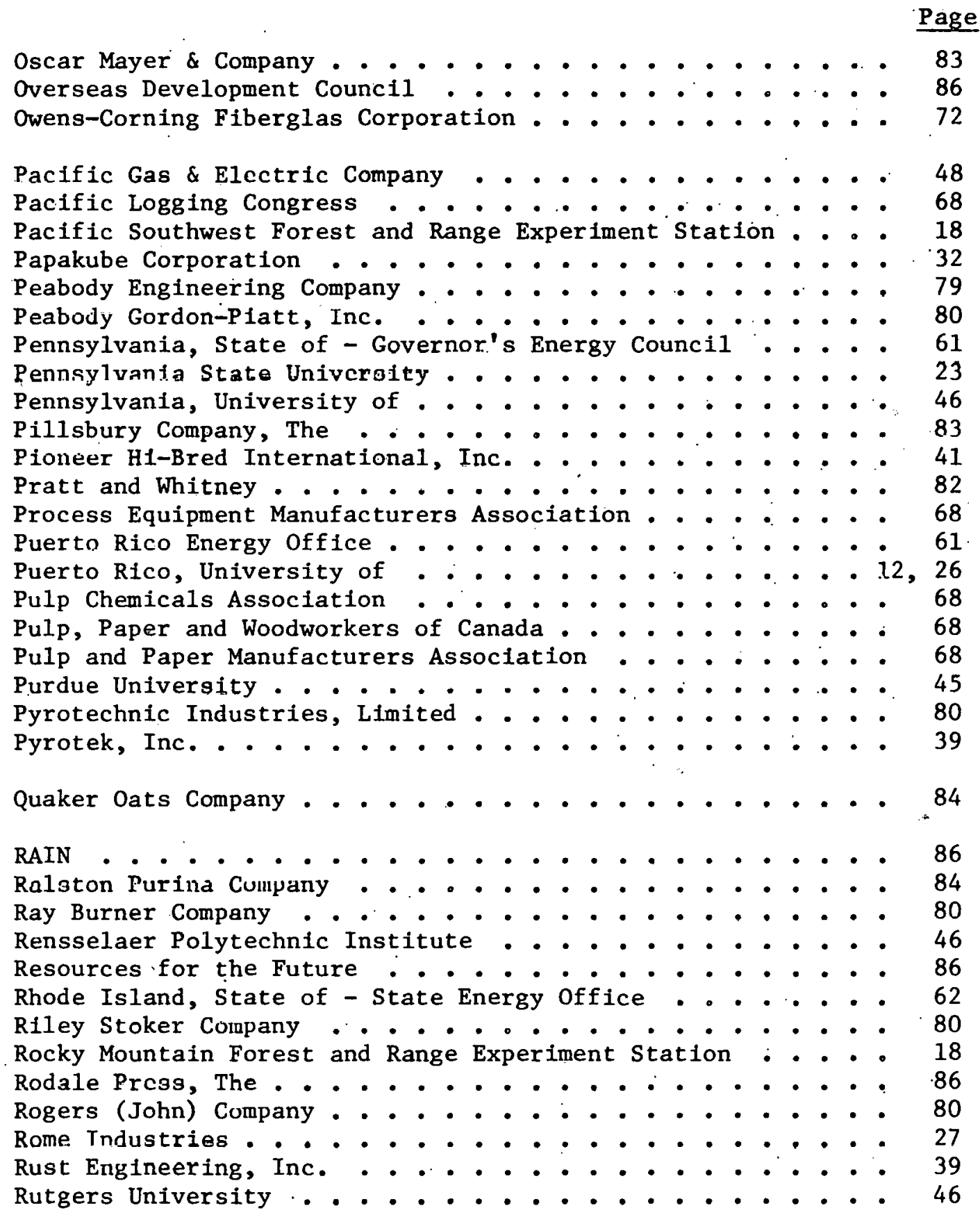


Science Applications, Inc. . . . . . . . . . . . . 31

Scott Paper Company :. . . . . . . . . . . . 73

Seattle Boiler Company ................. . 80

Seattle City Light Company . . . . . . . . . . . . 24

Sheaffer and Roland, Inc. . . . . . . . . . . 48

Sierra Club ...................... 87

Smith (Perry) Company, Inc. .............. 80

Society of Wood Science and Technology . . . . . . . . 68

Solar Energy Research Institute . . . . . . 5, 12, 24, 45

Sonoco Products .................... 37

South Carolina Commission on Forestry . . . . . . . . 23

South Carolina, State of - Energy Management Office . . . . 62

South Dakota, State of - Energy Policy Office . . . . . . 62

Southeastern Forest Experiment Station . . . . . . . . 18

Southern California Edison Company . . . . . . . . . 70

Southern California Gas Company .............. 48

Southern Forest Products Assoclation . . . . . . . . . 68

Southern Forest Experiment Station... . . . . . . 18

Sprout Waldron Company, Inc. . . . . . . . . . . . . 34

Stanford Research Institute. . . . . . . . . . . 19

Stanford University ................... . . 48

State, Department of - International Energy R\&D . . . . . 53

Stearns-Roger, Inc. . . . . . . ........... 80

Steel Craft Corporation .. . . . ........... . 80

St. Regis Paper Company ................. 73

J. P. Stevens . . . . . . . . . . . . . . . . 82

TechAir Corporation . . . . . . . . . . . . 41

Technical Association of the Pulp and Paper Industry . . . 68

TEKNEKRON ..................... 15

Tennessee, State of - Tennessee Energy Office . . . . . . 62

Tennessee Valley Authority . . . . . . . . . . . .12, 37

Texas, State of - Assistant to the Governor for Energy . : . 62

Resources

Texas Technical University .............. . . 41

Textile Workers Union of America .. . . . . . . . . 82

Timber Engineering Company ............... 73

Timber Products Manufacturers . . . . . . ..... 69

Toronto, University of ................. 46

Transportation, Department of ............. 54

U1trasystems, Inc. . . . . . . . . . . . . . 37

Union Carbide Corporation . . . . . . . . . . . .41, 74

United Gas and Pipeline Company ............. 70 
INDEX (Concluded)

\begin{abstract}
$\underline{\text { Page }}$
United Nations . . . . . . . . . . . . 88

United States Information Agency . . . . . . . . . . 54

United Technologiés . . . . . . . . . . . . . . 82

United Technologies Research Center . . . . . . . . 15

U.S. Department of Agriculture

Agricultural Experiment Station $. .28,25,28,49,50,52,54$

Agricultural Research Service ......., 20, 25, 50

Beltsville Agricultural Research Center . . . . . . 52

Forest Service....... 16, 17, 18, 25, 26, 27, 43, 54

Utah, State of - Department of Natural Resources . . . . 62

Utah State University :. . . . . . . . . . . 26

Vermont Agency of Environmental Conservation . . . . . . 38

Vermont National Resources Council . . . . . . . . . . 38

Vermont, State of - State Energy Office . . . . . . . 63

Virginia Polytechnic Institute . . . . . . . . . . 26

Virginia State Forestry Commission . . . . . . . . 26

Virginia, State of - Virginia Energy Office . . . . . . 63

Virginia, University of .............. 39

Virgin Islands Energy office . . . . . . . . . . 63

Vogt (Henry) Machine Company . . . . . . . . . . 81

Volunteers in Technical Assistance .. . . . . . . 87

Washington, State of - Washington Energy Office . . . . 63

Washington State University ............. 26

Washington, University of . . . . . . . . . . . . 25

WESTVACO Research Center . . . . . . . . . . . . 16

West Virginia, State of - Fuel and Energy Office . . . . 63

West Virginia, University of . . . . . . . . . . 41

Weyerhaueser Company ............ . 16,41, 73

Wheelabrator Clean Fuels Corporation . . . . . . . . 38

Wheelabrator-Frye, Inc. ... ............. 38

Wisconsin, State of - Office of State Planning and Energy : 63

Woodamation, Inc. ............... 38,81

Woods Hole Oceanographic Institute . . . . . . . . 32

Worldwatch Institutè . . . . . . . . . . . 87

Wright-Malta Corporation.............. 42

Wyatt Engineers, Inc. . . . . . . . . . . . 81

Wynming, State of - Department of Economic Planning . . . 64

Development
\end{abstract}

York-Shipley, Inc. . . . . . . . . . . . . 81

Zurn Industries, Inc. . . . . . . . . . . . 81 